\title{
MECHANICALLY LOADED EX VIVO BONE CULTURE SYSTEM 'ZETOS’: SYSTEMS AND CULTURE PREPARATION
}

\author{
CM Davies ${ }^{1}$, DB Jones ${ }^{2}$, MJ Stoddart ${ }^{1}, \mathrm{~K}$ Koller ${ }^{1}, \mathrm{E} \mathrm{Smith}^{4}, \mathrm{CW}$ Archer $^{3}$ and RG Richards ${ }^{1 *}$ \\ ${ }^{1}$ AO Research Institute, AO Foundation, Davos, Switzerland; ${ }^{2}$ Experimental Orthopaedics \& Biomechanics, \\ Philipps University Marburg, Germany; ${ }^{3}$ Cardiff School of Biosciences, Cardiff University, Wales, UK; ${ }^{4}$ University \\ of Wisconsin Medical School, Madison, Wisconsin, USA
}

\begin{abstract}
This paper introduces the culture preparation of ovine, bovine and human cancellous bone cores to be used in an explants model Zetos. The three dimensional (3D) bone cores were prepared and evaluated for all three animals. Bone cells in vivo constantly interact with each other, migratory cells, surrounding extracellular matrix (ECM) and interstitial fluid in a microenvironment, which continuously responds to various endogenous and exogenous stimuli. The Zetos system was designed to culture and mechanically load viable cancellous bone explants in their near natural microenvironment. This 3D ex vivo system bridges the current gap between in vitro and in vivo methods. One aim of this work was to compare the macro and micro-architecture of ovine, bovine and human cancellous bone tissue in preparation for culture within the Zetos system in order to determine the optimal source of experimental material. A second aim was to optimise the preparations of the bone cores as well as develop techniques involved during tissue maintenance. Bone core response was visualised using histological and immunohistochemical methods. The results demonstrate that cancellous bone explants vary greatly in trabecular density and bone volume depending on species, age and location. Sheep and human samples displayed the greatest variation between bones cores when compared to bovine. Even cores taken from the same animal possessed very different characteristics. The histology demonstrated normal bone and cell structure after the core preparation. Immunohistochemistry results demonstrated antigen retention after preparation methods.
\end{abstract}

Key Words: Bone, culture, histology, immunohistochemistry, species variation, bioreactor, Technovit

*Address for correspondence:

RG Richards,

AO Research Institute,

AO Foundation,

Clavadelerstrasse 8 ,

CH7270 Davos Platz,

Switzerland.

Tel: +41 (0)814142397

E-mail:geoff.richards@aofoundation.org

\section{Introduction}

In vivo bone research has the potential to gain insight into growth and development, systemic response to varied cytokines, growth factors, and fracture repair of bone. In vitro bone research has three main branches: i) Cell culture - the growth of cells no longer organised into tissues in vitro, where cells are harvested from tissue either mechanically or through enzyme digestion and propagated in a cell suspension or attached to a surface as a monolayer. ii) Tissue culture - the maintenance of tissue fragments in vitro, not necessarily preserving tissue architecture. iii) Organ culture - the maintenance or growth of tissue, or organs (in whole or in part) in vitro, which may allow differentiation and preservation of architecture and function. Advantages of using cell culture include the great number of molecular and biochemical tools currently available and experimental replicates can be virtually identical, making statistical analysis and quantification easier. However, cells may suffer from phenotypic drift, which may be due to dissociation of cells from their threedimensional geometry and their propagation on a twodimensional substrate. The dynamic properties of cell culture are difficult to control in vitro and it is also difficult to recreate the appropriate cell interaction found in vivo. Organ culture of cartilaginous tissue (Strangeways and Fell, 1926; Fell and Robinson, 1929) was developed to preserve the original structure and function of the tissue with regard to structural relationships of one cell type to others of the same or different lineage. However, since systemic factors are absent, the extrapolations to the in vivo situation were limited. Biochemical and molecular techniques in organ cultures are limited because the multicellular populations makes determination of specific biochemical pathways attributed to a specific cell difficult. Organ cultures have a short lifespan, as they often undergo central necrosis due to vascular occlusion and rate-limiting mass transfer. The loss of the vascular system has implications in limiting the size of organ sample that can be harvested, since cells in culture depend upon diffusion of nutrients and metabolites as well as for the removal of waste. Proliferation may thus be limited to the outer cell layer while necrosis may occur in the centre of the explants. Many researchers use non-physiological oxygen partial pressure (Lozupone et al., 1996; Meghji et al., 2001), which also affects the maintenance of the tissue.

One fundamental limitation to skeletal tissue culture is the lack of controlled physiological loading; which is known to induce extracellular cell matrix (ECM) production. It is hypothesised that osteocytes sense changes in mechanical loading within their lacuna- 
canalicular environment and transduce this information to produce a change in bone architecture. Without load, bone will have a net increase in resorption, as is seen in patients after prolonged bed rest (Vico et al., 1987) or astronauts in zero gravity (Spengler et al., 1983). The limitations of current in vitro loading experiments are often that they are performed on tissue explants or extracted cells that do not mimic the in vivo environment. Loading conditions of the tissue are often not calibrated or quantified and do not mimic physiologic condition, for example many load studies involve the use of calvarial cells even though it is known they behave very differently to cells derived from load bearing limbs (Rawlinson, 1995). The effects of loading of single cells or cell monolayers are difficult to extrapolate to the in vivo situation due to the lack of a 3D matrix. Deformation of cells through bending of culture substrate can cause a series of undesirable effects such as stimulation through fluid flow or shear stresses. It is also essential to measure all parameters as frequency rate, and magnitude of load will all affect cellular responses. To study the processes whereby skeletal tissue reacts to mechanical stresses, experimental systems are needed that allow direct measurement of cell proliferation, differentiation, metabolism and matrix formation under controlled loading conditions. For bone research to be conducted that closely resembles the in vivo $3 \mathrm{D}$ environment it is imperative to use a culture system that allows bone explants to receive and measure physiological loads.

A novel ex vivo mechanically loading culture system for 3D cancellous bone tissue (fig 1 and 2) has been developed to overcome some of the limitations discussed above for both cell culture and organ culture (Jones et al., 2003). Force and/or compression can be applied and measured in real time with high precision $(+/-3 \%)$ during the application of a variety of different wave forms and frequencies which mimic in vivo conditions. The physiochemical environment can be monitored and controlled. The system has potential in pre-testing the integration of bone with biomaterials, studying basic bone biology, bone diseases and defects e.g. in osteoporosis.

Herein we describe tissue preparation and evaluation for the culture of ovine, bovine and human tissue for the Zetos system. Discussed will be aspects including the optimal source of tissue for experimentation, media diffusion rate analysis and the effect of tissue harvesting on sample parallelism and bone debris. The aim of this work is to develop a reproducible, sterile method for producing large numbers of living bone cores for use in biomechanical studies. We also sought to characterise the tissue from different sources using several methods. We also will describe histology and immunohistochemistry analysis of the bone cores after undergoing biopsy and preparation for culture.

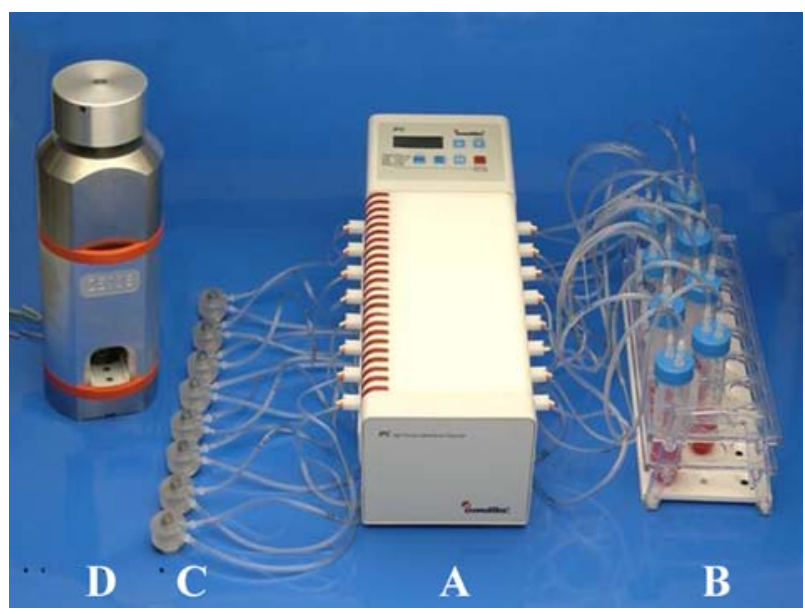

Figure 1. Ex vivo bone loading culture system. Microprocessor controlled low pulsation dispensing pump (A) allows diffusion of fresh media (B) through the bone cores within the chambers $(C)$. The bone cores are stimulated daily within the 'Zetos' computer controlled mechanical loading device (D). The device is $30 \mathrm{~cm}$ high, made of stainless steel surrounding a piezoelectric stack linked to a voltage amplifier to enable expansion and contraction to apply and remove force onto the chamber piston and bone core. There is a preload knob on the top to place a force onto each piston. It has an opening for insertion of the individual chambers (front, bottom) daily loading of the bone cores. Three metal rods inside this opening ensure that the chambers are always inserted correctly.
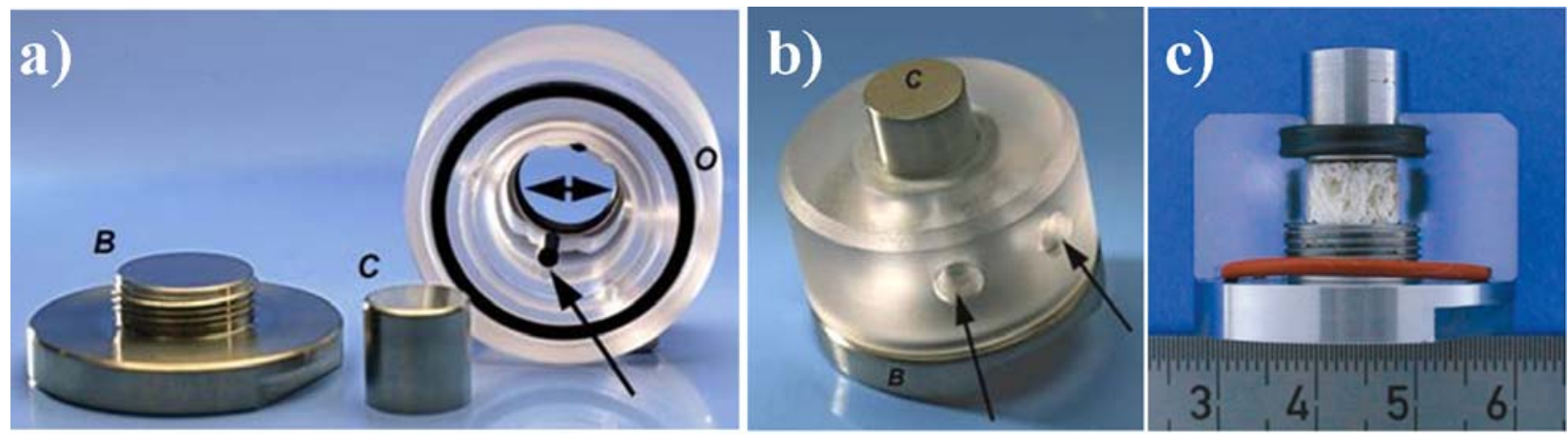

Figure 2. a) Stainless steel platen base (B). On top of the chamber, when in use, there is a stainless steel platen cylinder (C) sealed by an X-ring (double headed arrow) which also provides some flexibility to minimize tilting of the piston around the vertical axis. The polycarbonate chamber has an entry and exit port to allow media to enter and leave the chamber (fig 2b). An O-ring (O) at the bottom of the chamber seals it to the base plate (B). The bone core is cushioned by two gaskets at the centre (arrow) that were designed to force the media through the bone (and not flow around the periphery). b) Constructed chamber, depicting the location of base plate (B), cylinder (C) and media exit and entry points. c) Cross section through the assembled chamber complete with bone core. 


\section{Materials and Methods}

\section{Bone Maceration}

Bone maceration was used to show the general structure of bone core used in the Zetos system of ovine (femora), bovine (metacarpals) and human tissue (human femoral heads). Two limbs from each species (except human) were assessed in the following manner: 1) samples were obtained and cleaned to remove as much soft tissue as possible. 2) A hole was drilled in through the bone in order to allow all the marrow to be washed out with water, or blown out with an air gun. 3) The bones were boiled in water for 5 $15 \mathrm{~min}$ in order to denature the proteins. The bones were then placed for several days in a $1 \%$ solution of papain (Fluka 76222 ) at $65^{\circ} \mathrm{C}$, which was changed twice a week to remove all the cellular material; this was continued until the solution was clear. The tissue was then washed for $24 \mathrm{~h}$ under running water and a brush was used to remove any remaining tissue. A $3.5 \%$ solution of hydrogen peroxide (Fluka 95299) was used to deactivate the papain by boiling the bones for $1-2 \mathrm{~h}$, followed by a brief wash under running water and air dried. The fat was removed from the tissue by placing the bones in chloroform (Fluka, 25669 ) for $24 \mathrm{~h}$ and then the samples were left to air dry.

\section{Harvesting Cancellous Bone Cores}

Cancellous bone explants were obtained either from ovine distal femora (3-6 years of age), bovine distal metacarpals (4 months of age) or human femoral heads (obtained from a 70 year old female, an 80 year old female and one 71 year old male during total hip replacement) (Ethic Commission Graubünden approval 18/02). The skin, muscle, and tissue were removed from ovine and the bovine bone under sterile conditions, in a laminar flow hood. The bone tissue was cut into $9 \mathrm{~mm}$ thick sections with an Exakt 300 (Exakt Apparatebau GmbH \& Co. KG, $\mathrm{DE}$ ) band saw (fig 3a). Cores, $10 \mathrm{~mm}$ in diameter, were drilled from the sections with a specially designed diamond tipped, hollow, fluted, drill (D181) (fig 3b). The cores were cut parallel with either the Exakt band saw or a Leica annular saw (Leica AG, Glattbrugg, $\mathrm{CH}$ ) to the height of 5 $\mathrm{mm}$ (fig $3 \mathrm{c} \& \mathrm{~d}$ ). Throughout all cutting procedures, the bone was irrigated with sterile $0.9 \% \mathrm{NaCl}$ at $4{ }^{\circ} \mathrm{C}$ (Fresenius Kabi NI2516) to help remove bone debris, and to limit the amount of damage caused to the cells by heat. All surfaces and machines were cleaned with $70 \%$ ethanol and antibacterial fluids $24 \mathrm{~h}$ and $12 \mathrm{~h}$ before the cutting process. After harvesting the bone, cores required washing in order to remove as much bone dust and debris as possible, which may clog soft tissue or pores. The cores were placed in $12 \mathrm{ml}$ of Hank's Balanced Salt Solution (HBSS) and then were then washed $3 \times 10 \mathrm{~min}$ with $\mathrm{HBSS}$ at $37^{\circ} \mathrm{C}$. A final wash of HBSS containing 100,000 IU Penicillin/ Streptomycin, $500 \mu \mathrm{g}$ Fungizone and $150 \mathrm{mg}$ Gentamicin per $\mathrm{L}$ for $30 \mathrm{~min}$ was performed before the bone cores were inserted into the Zetos culture chambers. The washing procedure aims to accomplish two things: 1) wash out the drill residue without disturbing the marrow and bone lining cells and 2) a higher level of antibiotics will help to reduce the potential for specimen contamination. At least two bone
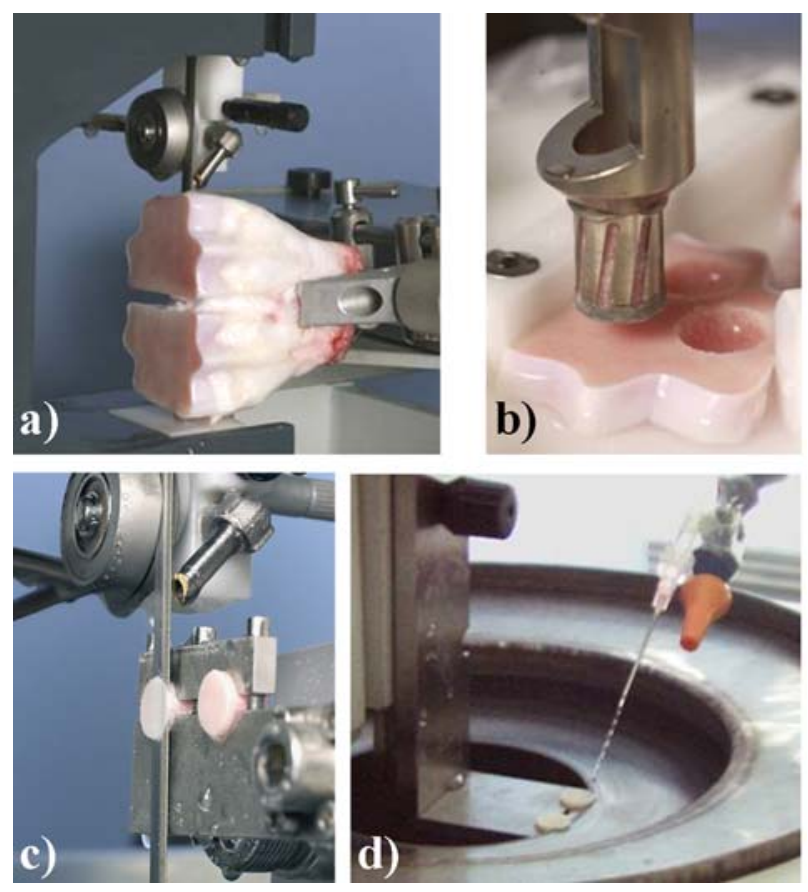

Figure 3. Bone preparation. During all preparation procedures bone was irrigated with sterile saline. a) The Exakt 300 band saw with bovine metacarpal, 200 $\mu \mathrm{m}$ thick diamond-coated blade. b) The bone sections (bovine metacarpal bone) were clamped into a Teflon holder on an x-y milling machine (Eco MAC 212). The chuck contained a specially crafted drill bit with a diamond-coated cutting surface. c) By inserting a selfmade holder onto the sliding arm of the Exakt 300 band saw it was possible to hold two bone cores and cut them roughly parallel to the desired height of $5 \mathrm{~mm}$. d) The Leica annular saw, with self-made mounting apparatus, was also used to cut the cores parallel to 5 mm height.

cores per preparation were fixed in $70 \%$ ethanol as soon as harvest procedure was complete as baseline controls (T0).

\section{Radiology}

Ovine distal femora, bovine distal metacarpals and human femoral heads were cut into $7 \mathrm{~mm}$ thick sections with the Exakt 300 band saw. The samples were placed on photographic film (AGFA Structurix D4 film) and exposed to $35 \mathrm{kV}$ for $2 \frac{1}{2} \mathrm{~min}$ in a Faxitron 804 (Faxitron Company, Illinois, USA).

\section{Micro-computed Tomography $(\boldsymbol{\mu C T})$}

Cores were harvested (Ovine 17 cores, Bovine 18 cores, Human 33 cores), placed in $5 \mathrm{ml}$ of $0.9 \% \mathrm{NaCl}$ and stored at $-20^{\circ} \mathrm{C}$. Samples were defrosted before being measured with the MicroCT 20 (Scanco Medical AG, Bassersdorf, $\mathrm{CH})$. Voxel size of $26 \times 26 \times 26 \mu \mathrm{m}^{3}$ was used. For the evaluation procedure, a VOI (volume of interest), which was smaller than the explant was chosen to eliminate debris produced from drilling. The VOI was a cylinder with a diameter of $8.944 \mathrm{~mm}$ and a height of $4.888 \mathrm{~mm}$. The chosen segmentation parameters were sigma 1.0, support 
2 and threshold 250. Sigma and support are the parameters of the Gauss filter. These parameters were instrument specific.

\section{Evaluation of surface topography of cut bone cores} Bone cores were left to air-dry for $24 \mathrm{~h}$ before the bone surfaces, cut with either the EXAKT saw or annular saw were measured with a FRT MicroProf non-contact profilometer (Fries Research \& Technology $\mathrm{GmbH}$, Bergisch Galdbach, Germany). Three random measurements were taken per surface, and the roughness (Ra) was measured from a $1 \mathrm{~mm} \times 1 \mathrm{~mm}$ analysis area scan at a speed of $0.1 \mathrm{~mm} / \mathrm{s}$ with a measurement rate of 100 points per second.

\section{Viability assessment using Calcein AM \& Ethidium homodimer 1.}

Bone cores were excised as described above washed $3 \mathrm{x}$ $30 \mathrm{~min}$ in $10 \mathrm{ml}$ sterile PBS then incubated with $1 \mu \mathrm{g} / \mathrm{ml}$ Calcein AM (hydrolysed within a cell to calcein, which is a polyanionic derivative of fluorescein. Calcein AM is an uncharged molecule that can permeate cell membranes and is cleaved by esterases in the cell cytoplasm, detecting living cells) and $1 \mu \mathrm{g} / \mathrm{ml} \mathrm{EthD-1} \mathrm{(a} \mathrm{cationic,} \mathrm{cell-}$ impermeant, red fluorescent dye to detect dead or dying cells. It binds to DNA causing a 40 times enhancement of the fluorescence) in sterile PBS. Bone cores were placed in $4 \mathrm{ml}$ of staining solution and incubated at $37^{\circ} \mathrm{C}$ for $2 \mathrm{~h}$ in the dark. After incubation, the cores were washed in sterile PBS. The cores were imaged on the Zeiss photomicroscope (Axioplan Imaging) and processed with Axiocam and Axiovision.

\section{Bone core diffusion vs perfusion tests}

Freshly harvested bovine bone cores were placed in the Zetos culture chambers at $37^{\circ} \mathrm{C}$. Dulbecco's Modified Eagle Media (DMEM) $+10 \%$ Foetal Calf Serum (FCS), or $10 \%$ disulphine blue (Merck, VN150) or $10 \%$ procion red (BASF, H8BN) was perfused for $3 \mathrm{~h}$ at a flow rates of 7,15 , or $35 \mathrm{ml} / \mathrm{h}$. Bone cores were prepared (as previously described) and immediately imaged with a digital camera (Minolta) fitted with a Macro lens. A perfusion holding system for the bone core was designed to determine the pressure required to induce perfusion through the bone core. The bone core was inserted in a silicon tube, to prevent liquid flow around the sides of the bone core a cable binder was tightened around the tube pressing it against the bone core (fig $4 \mathrm{a}$ ). The liquid under the bone was collected in a glass beaker (fig $4 b$ ) which was weighed before and after the run to get the mass of liquid going through the bone. Knowing the specific gravity of the liquid and the height of the liquid column the pressure over the bone can be calculated using following formula:

$$
\mathrm{P}=\mathrm{r} \cdot \mathrm{g} \cdot \mathrm{h}
$$

Where $\mathrm{P}=$ pressure, $\mathrm{r}=$ specific gravity, $\mathrm{g}=$ gravity constant $(9,81 \mathrm{~N} / \mathrm{kg}), \mathrm{h}=$ height of the liquid over bone.

\section{Fixation and embedding}

Bone cores of $5 \mathrm{~mm}$ thickness and a diameter of $10 \mathrm{~mm}$ were fixed with $70 \%$ ethanol, for five days, at $4^{\circ} \mathrm{C}$ with fresh changes daily. Bone cores were then sawn in half with the Exakt 300 band saw to give a core with a radius of $5 \mathrm{~mm}$ and a height of $5 \mathrm{~mm}$. Samples were placed in fresh $70 \%$ ethanol at $4{ }^{\circ} \mathrm{C}$ for a further $24 \mathrm{~h}$. The cores were dehydrated in an ethanol series $(80 \%, 96 \%, 100 \%, 100 \%)$ for a day each and were cleared in xylene (Siegfried, $235120-04)$ for $12 \mathrm{~h}$ at room temperature $\left(20-22^{\circ} \mathrm{C}\right)$. The bone cores were then embedded in light protective jars, as described by Yang et al., 2003, in Technovit 9100 New ${ }^{\circledR}$ (Heraeus Kulzer GmbH, Germany). Briefly samples were pre-infiltrated in Technovit solutions 1-3 for 1 day each at $4^{\circ} \mathrm{C}$, before being transferred to solution 4 . Samples were left to infiltrate in solution 4 for 5 days at $4^{\circ} \mathrm{C}$ before being polymerized in solution 5 at $-20^{\circ} \mathrm{C}$ to retain sample antigenicity. The jar used to polymerise the sample was filled completely with solution 5 to exclude any air, therefore improving the polymerisation. Solution infiltration times are determined by sample size, larger samples requiring longer infiltration times. The embedded bone cores usually polymerised within 5-10 days. One sample was then opened to ensure complete polymerisation had occurred, if not polymerisation was allowed to proceed for a further 7 days. One half of each core was orientated to display the cross-section while the other half was orientated to give the transverse-section. Polymerised cores were taken from $-20^{\circ} \mathrm{C}$ and placed at $4^{\circ} \mathrm{C}$ overnight. Cores were then brought to room temperature and sectioned with the HM355S rotary microtome (Microm International) with a D blade to produce $6 \mu \mathrm{m}$ thick sections. Sections were adhered to SuperFrost ${ }^{\circledR}$ Plus (Roth, Karlsruhe, D, no. H867) glass slides with $80 \%$ ethanol and covered with polyethylene foil and paper before being clamped for $24 \mathrm{~h}$ at $50^{\circ} \mathrm{C}$.

Prior to staining, the residual resin was removed from the tissue sections by placing the glass slide in MEA (1acetoxy-2-methoxy-ethane) (Fluka, 00860) for 2 x $30 \mathrm{~min}$, then through a graded ethanol series $(100 \%, 96 \%)$ before being placed in distilled water ready for morphological staining. The following stains were used for morphology.

Masson-Goldner Trichrome: Samples were placed in Weigert's haematoxylin (Merck, 1.15973./1 and 1.15973./ 2) stain for $1 \mathrm{~h}$, rinsed under lukewarm water for $5 \mathrm{~min}$, immersed in Masson solution (Fluka, 84600, and 81465) for $15 \mathrm{~min}$, differentiated in 1\% acetic acid (fresh) (Fluka, 45731) before being placed in phosphomolybdic acid orange G (Fluka, 79560, and Fluka, 75380) for $8 \mathrm{~min}$. Samples were rinsed in $1 \%$ acetic acid, immersed in $0.1 \%$ Light green SF yellowish (Merck, 15941) for $12 \mathrm{~min}$, rinsed in 1\% acetic acid then de-ionised water, 96\% ethanol, absolute ethanol ( $2 \times 2 \mathrm{~min})$, rinsed in xylene and then immersed in xylene for $5 \mathrm{~min}$ and mounted with Eukitt (O. Kindler GmbH \& Co., Freiburg, Germany).

Toluidine blue: - Samples were stained with 1\% Toluidine blue for $30 \mathrm{~min}$, rinsed in water followed by immersion in 96\% ethanol for 5-10 seconds, rinsed in absolute ethanol $(2 \times 3 \mathrm{~min})$, rinsed in xylene for $5 \mathrm{~min}$ and mounted with Eukitt.

Giemsa:- Samples were immersed in 15\% Giemsa (Fluka, 48900) for $40 \mathrm{~min}$, rinsed for a few seconds in water to 
check staining intensity, differentiated in $96 \%$ ethanol for $1 \mathrm{~min}$ before being placed in $100 \%$ ethanol for $5 \mathrm{~min}$. Sections were rinsed in xylene for $5 \mathrm{~min}$ and mounted with Eukitt.

Movat Pentachrome: Samples were stained with alcian blue (Fluka, 05500) for $10 \mathrm{~min}$, then rinsed in running water for $6 \mathrm{~min}$. Sections were then immersed in alkaline alcohol (Siegfried, 106440-01) for $60 \mathrm{~min}$, then rinsed in running water for $10 \mathrm{~min}$. Next, sections were stained with Weigert's haematoxylin (Merck, 1.15973./1 and 1.15973./ 2) stain for $20 \mathrm{~min}$, rinsed under lukewarm water for 15 min, and then differentiated in $96 \%$ ethanol. Sections were rinsed in $\mathrm{dH}_{2} \mathrm{O}$ before being immersed in brilliant crocein/ fuchsine acid for $20 \mathrm{~min}$. Sections were rinsed in $0.5 \%$ acetic acid before being stained with $5 \%$ phosphotungstic acid (Fluka, 79690) for $20 \mathrm{~min}$. Sections were then rinsed in $0.5 \%$ acetic acid before being stained with Saffron alcohol for $40 \mathrm{~min}$. Finally, sections were washed three times in $100 \%$ ethanol, $5 \mathrm{~min}$ in each change of ethanol before rinsed in xylene for $5 \mathrm{~min}$ and mounted with Eukitt.

\section{Immunohistochemistry}

Antibodies: Polyclonal rabbit antibodies against human osteopontin (OPN) was a generous gift from Dr. Larry W. Fisher of the National Institutes of Health, National Institute of Dental and Craniofacial Research, Craniofacial and Skeletal Diseases Branch, Bethesda, MD, USA: Mouse immunoglobulins (IgG) were purchased from Sigma (St. Louis, MO, USA) (I8765).

Immunolabelling: Sections were labelled for osteopontin as described by Yang et al. 2003. Briefly, the immunolabelling procedure was as follows: Sections were blocked with $6 \%$ goat serum. Primary rabbit anti-human osteopontin antibodies $(3.3 \mu \mathrm{g} / \mathrm{ml})$ were incubated for $1 \mathrm{~h}$ at $21^{\circ} \mathrm{C}$. Sections were immunolabelled for $2 \mathrm{~h}$ at room temperature with a $5 \mathrm{~nm}$ gold-conjugated goat anti-mouse secondary antibody $(1: 200)$. Sections were washed 6 times before being incubated with $1 \%$ glutaraldehyde in PIPES $\mathrm{pH} 7.4$ for 5 min to prevent the gold conjugates dissociating, followed by being washed 3 times with PIPES and rinsing with distilled water. Sections were silver enhanced (silver enhancement kit, British Biocell International, Cardiff, UK) for $30 \mathrm{~min}$. The reaction was stopped by placing the slides in distilled water for $5 \mathrm{~min}$. No counterstaining was used. Slides were dehydrated through a graded series of ethanol, cleared in xylene and Eukitt-mounted.

\section{Results}

\section{Bone Maceration}

The maceration of the tissue allowed the samples to be preserved and the trabecular structure and orientation plus the shape of the bone as a whole to be viewed without the presence of soft tissue (fig 5). In all three species the trabeculae became more sparse as the medullary cavity was approached. The internal structure of the ovine femora (fig 5a) is small and only a few bone cores could be harvested from the femoral head, unlike that of the human
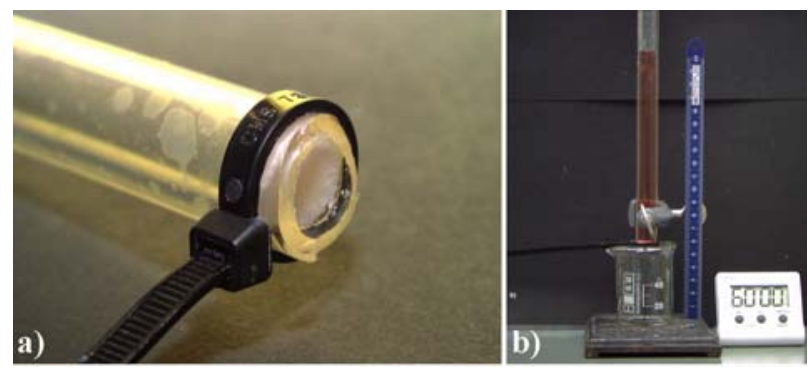

Figure 4. a) Bone core inserted in a silicon tube filled with DMEM with a cable binder around the tube. b) The liquid under the bone should have been collected in a glass beaker, but none emerged.

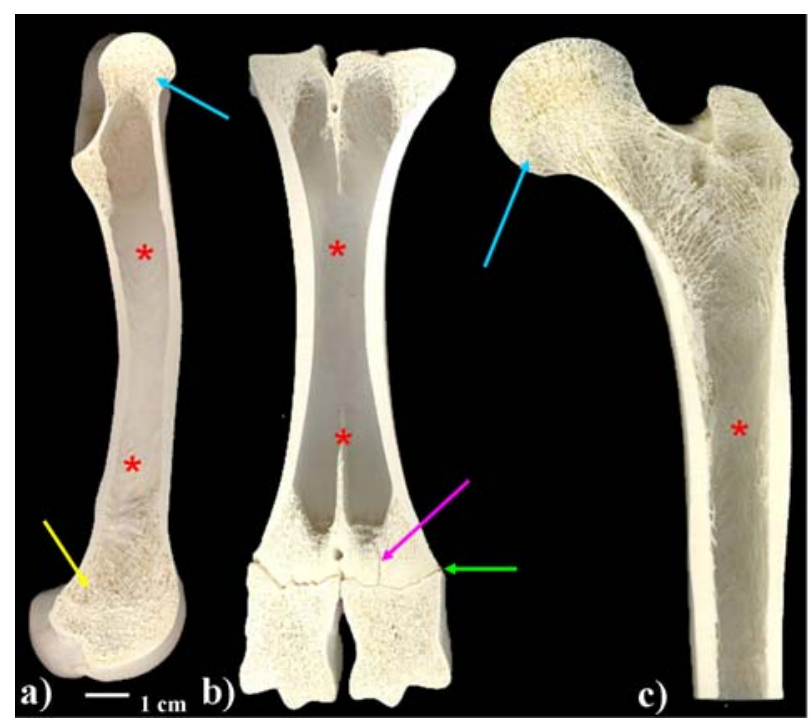

Figure 5. Planes of the system models all showing thinning of the cancellous tissue as it approaches the marrow cavity of the diaphysis (red star). a) Femora (transverse plane) of a 4-year-old ewe. The femoral head (blue arrow) is too small to harvest tissue from, there being more cancellous tissue in the distal region (yellow arrow). b) A four month old calf metacarpal (transverse plane) displaying the distal condyle (green arrow) and a fracture above the growth plate (pink arrow). c) The femur of human (frontal/coronal plane). It is possible to see the orientation of the trabeculae. There is plenty of cancellous bone for samples available within the femoral head (blue arrow).

femoral head (fig 5c). The growth plate can be seen at the distal region of the bovine metacarpals (fig $5 b$ ). Just above the growth plate a fracture was observed. It is common for calves to fracture their metacarpals as this bone supports much of the animals' weight.

\section{Radiology of tissue.}

Analysis of the structure of cancellous bone demonstrated various architecture within the same animal, between different animals and between the different species. Radiograph images of $7 \mathrm{~mm}$ thick bone segments allowed qualitative observations of cancellous bone density to be made. It was apparent after analysis of the right and left femora from 11 female sheep that ranged from 3-5 years of age and 40-63 kg in weight that these animals often 


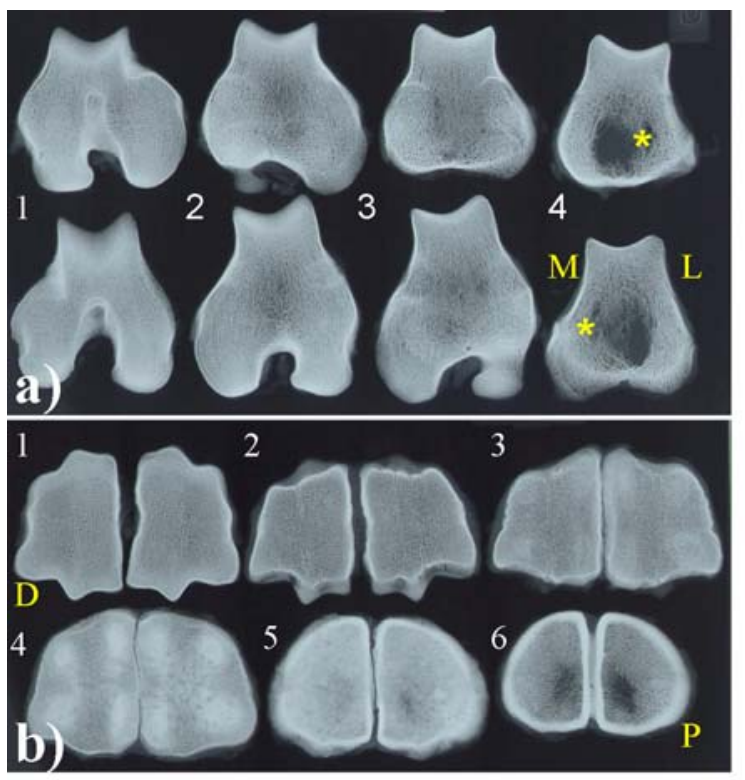

Figure 6. Radiographs taken at $35 \mathrm{kV}$ for $2 \frac{1}{2} \mathrm{~min}$. Anatomical orientation; $\mathrm{M}=$ medial; $\mathrm{L}=$ lateral; $\mathrm{D}=$ distal; $\mathrm{P}=$ posterior. An asterisk represents a void/cyst within the tissue. a) Ovine distal femora (3 years, 49 $\mathrm{kg}$ ). The sections in the upper row were from the left leg, while the sections in the lower row were from the right leg. The first section was the most distal and closes to the articular cartilage, while the last section was the most proximal and closest to the medullary cavity. b) Bovine distal metacarpal (4 months). The sections in the upper row were the most distal and closest to the articular cartilage, while the lower section were the most proximal and closest to the medullary cavity. The lower left section is thorough the growth plate. c) Human femoral head from a female patient, 80 years of age. The first section is the most medial and the last section the most lateral. A number of cysts are present within the cancellous bone tissue.

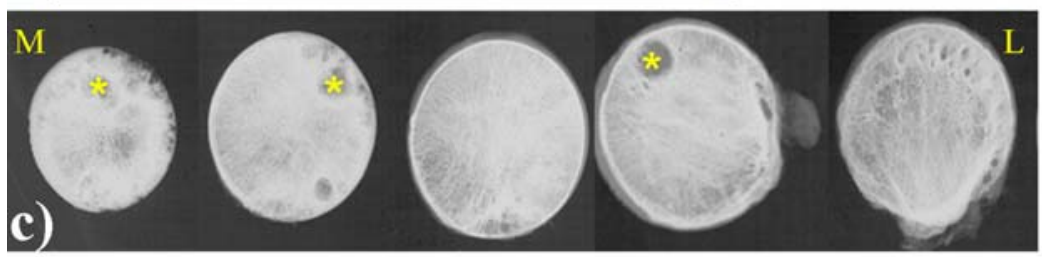

suffered from cysts within the cancellous tissue of the metaphysis (fig 6a). Cancellous tissue was always denser in the most distal section of bone, closest to the articular cartilage. Tissue became less dense in sections closest to the medullary cavity. On average three to four $9 \mathrm{~mm}$ bone slices could be obtained from each distal ovine femur, giving rise to approximately 15-19 cores per femur. Bovine metacarpal tissue (from 7 calves) was composed of a more homogeneous organisation of cancellous bone tissue, though after the first three slices the next two slices usually contained the growth plate, consisting of cartilage cells and ECM (fig 6b). The final bone slice usually lacked cancellous tissue due to the opening of the medullary cavity, thus only the first three slices were considered for culture in the Zetos system. On average it was possible to harvest approximately 24 quality cores from a single metacarpal. There was no indication of cysts present in any bovine sections from over 9 animals tested. The femoral heads from 3 human patients were observed by radiographs. The age of these ranged from 70-80 years. It was possible to obtain at least three bone slices from each head (fig 6c). The first slices (the most medial region) contained the densest amount of trabeculae, thereafter further towards the femoral neck the sections became less dense. Usually, the last slice contained very little cancellous bone. Humans are also known to develop cysts. These cysts were most apparent in the 80 or 81 -year-old human, where it was possible to observe voids. It may be beneficial to carry out radiological analysis prior to tissue harvest for the Zetos system in order to locate areas within the bone slices to avoid. However, the effect that making radiographs has on bone cells viability will have to be conducted and sterility of the method tested.

\section{MicroCT image of cancellous bone tissue}

The aim of making $\mu \mathrm{CT}$ analysis on individual bone cores was to show an appreciation that each sample (whether located adjacent to each other or far apart) differed in their micro-architecture. Thus, implying that comparison of one sample to another is not as simple as it is with more homogenous tissue such as cartilage. This technique provided a three-dimensional image of the cancellous bone explants. A smaller volume of interest than the actual explant was used, so that damage produced from drilling was not imaged which may have concealed the internal trabecular structure of the cores. It was also possible to obtain detailed histomorphometrical data regarding bone volume, bone surface area, trabecular thickness and number. Cores were analysed from within the cancellous tissue of i) ovine distal femora (fig 7a,b) of a 5-year-old ewe, which was $63 \mathrm{~kg}$ in weight (17 cores) ii) bovine distal metacarpals (fig 7c,d) of a 4-month-old calf (18 cores) and iii) human femora (fig 7e, f) 80 -year-old female patient (33 cores) Table 1. It was possible to see that each core was composed of a different number of plate and rod-like structures of ECM. It was apparent that there was a greater variance between bone volume within ovine and human tissue cores than within bovine tissue cores.

\section{Analysis of Core Preparation- Surface Parallelism}

The profilometer allowed qualitative analysis of the trabecular bone surface. Regular and parallel ridges were clearly seen on the calcified tissue that had been cut both with the EXAKT 300 band saw (fig 8a) and with the annular saw (fig 8b) which covered the entire surface. Analysis of the annular saw cut, showed a much lower roughness of the ridges. 


\begin{tabular}{|c|c|c|c|}
\hline Sample & Ovine & Bovine & Human \\
\hline Number of cores & 17 & 18 & 33 \\
\hline TV $\left[\mathrm{mm}^{3}\right]$ & 297.110 & 297.110 & 300.770 \\
\hline BV $\left[\mathrm{mm}^{3}\right]$ & $54.895 \pm 19.99$ & $59.888 \pm 12.98$ & $80.507 \pm 22.33$ \\
\hline BV/TV [1] & $0.185 \pm 0.07$ & $0.202 \pm 0.04$ & $0.268 \pm 0.07$ \\
\hline BS $\left[\mathrm{mm}^{2}\right]$ & $876.914 \pm 225.43$ & $1022.587 \pm 176.91$ & $808.292 \pm 143.52$ \\
\hline $\mathrm{BS} / \mathrm{BV}[1 / \mathrm{mm}]$ & $16.721 \pm 2.99$ & $17.212 \pm 1.09$ & $10.561 \pm 2.35$ \\
\hline SMI [1] & $0.606 \pm 0.59$ & $0.518 \pm 0.34$ & $0.583 \pm 0.75$ \\
\hline Tb.N [1/mm] & $1.476 \pm 0.38$ & $1.721 \pm 0.30$ & $1.344 \pm 0.24$ \\
\hline Tb.Th [mm] & $0.123 \pm 0.02$ & $0.117 \pm 0.01$ & $0.199 \pm 0.05$ \\
\hline Tb.Sp [mm] & $0.605 \pm 0.23$ & $0.480 \pm 0.10$ & $0.572 \pm 0.17$ \\
\hline \multicolumn{4}{|c|}{$\begin{array}{l}\text { TV = tissue volume; } \mathbf{B V}=\text { bone volume; } \mathbf{B V} / \mathbf{T V}=\text { bone volume to tissue } \\
\text { volume; } \mathbf{B S}=\text { bone surface; } \mathbf{B S} / \mathbf{B V}=\text { bone surface to volume ratio; } \mathbf{S M I}= \\
\text { structure model index; Tb.N = trabecular number; Tb.Th = trabecular thickness; } \\
\text { Tb.Sp = trabecular separation. }\end{array}$} \\
\hline
\end{tabular}

Table 1- Quantitative data obtained from $\mu \mathrm{CT}$ analysis of prepared bone cores. (Ave \pm S.D.)

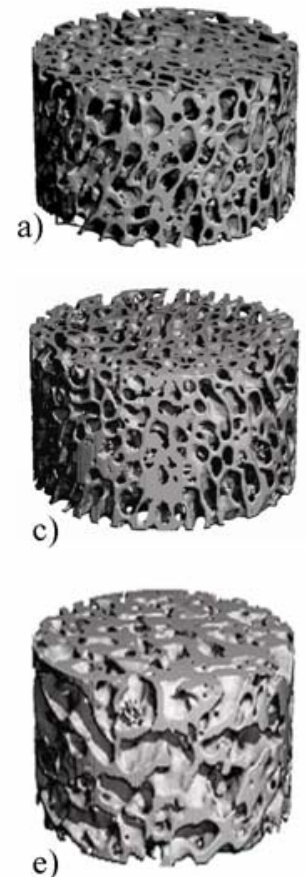

e) b)

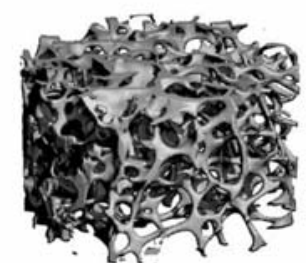

d)

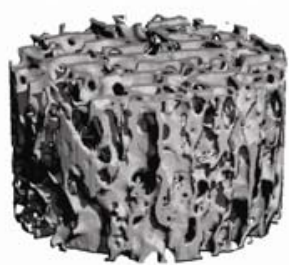

f)

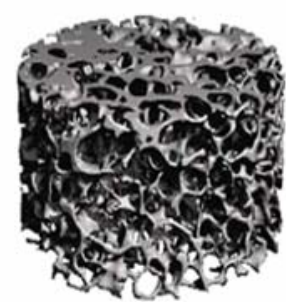

Figure 7. Architecture of bone cores harvested from the three species. a) Ovine core depicting the densest tissue explant from the medial side of the first section cut, (the most distal section). It has a bone volume of $91.8 \mathrm{~mm}^{3}$ from a total volume of $297.11 \mathrm{~mm}^{3}$. b) Ovine core depicting the least dense tissue explant from the centre of the second section (bone volume of $25.8 \mathrm{~mm}^{3}$ from a total volume of $297.11 \mathrm{~mm}^{3}$ ). c) Bovine core depicting the densest tissue explant from the first section cut, (most distal section). It had a bone volume of 95.3 $\mathrm{mm}^{3}$ from a total volume of $297.11 \mathrm{~mm}^{3}$. d) Bovine core depicting the least dense tissue explant from the third section cut (closest region to the growth plate). It had a bone volume of $45.6 \mathrm{~mm}^{3}$ from a total volume of $297.11 \mathrm{~mm}^{3}$. e) Human core depicting the densest tissue explant from the second section cut. It had a bone volume of $130.56 \mathrm{~mm}^{3}$ from a total volume of 300.77 $\mathrm{mm}^{3}$. f) Human core depicting the least dense tissue explant from the centre of the second section. It had a bone volume of $60.4 \mathrm{~mm}^{3}$ from a total volume of 300.77 $\mathrm{mm}^{3}$.

\section{Cell Viability after preparation of cores}

Calcein AM used in conjunction with Ethidium Homodimer 1 (EthD-1) demonstrated a layer of dead cells up to $1 \mathrm{~mm}$ in depth around the periphery of the bone cores (fig 8). It was possible to see the trabecular bone stained grey, the live marrow cells in between were stained green, and the dead cells, which were mainly seen on the periphery, were stained red. High power examination demonstrated that drilling of the cores killed the cells on the periphery of the bone core. The core surface area did not show as much cell death as there was at the periphery around the circumference of the cores. Fixing the above imaged bone cores in $70 \%$ ethanol at $4^{\circ} \mathrm{C}$ preserved the

labelling of the cells (fig $8 \mathrm{a}, \mathrm{b}$ ). However, the calcein stain did not remain within the cells through the embedding procedure, though EthD-1 did survive the process.

\section{Bone Core diffusion vs. Perfusion}

Disulphine Blue: After 3h of pumping media through the chambers which contained the bovine samples, complete core diffusion was not observed (fig 10a,b,c). On average, it was observed that the stain infiltrated approximately 2 $\mathrm{mm}$ from the exposed surface after $3 \mathrm{~h}$, independent of flow rate $(7,15,35 \mathrm{ml} / \mathrm{h})$. For some samples, the tight fit of the piston to the bone surface reduced stain diffusion (fig 10b). Figure $10 \mathrm{~d}$ is an example of a bone core diffused with the 

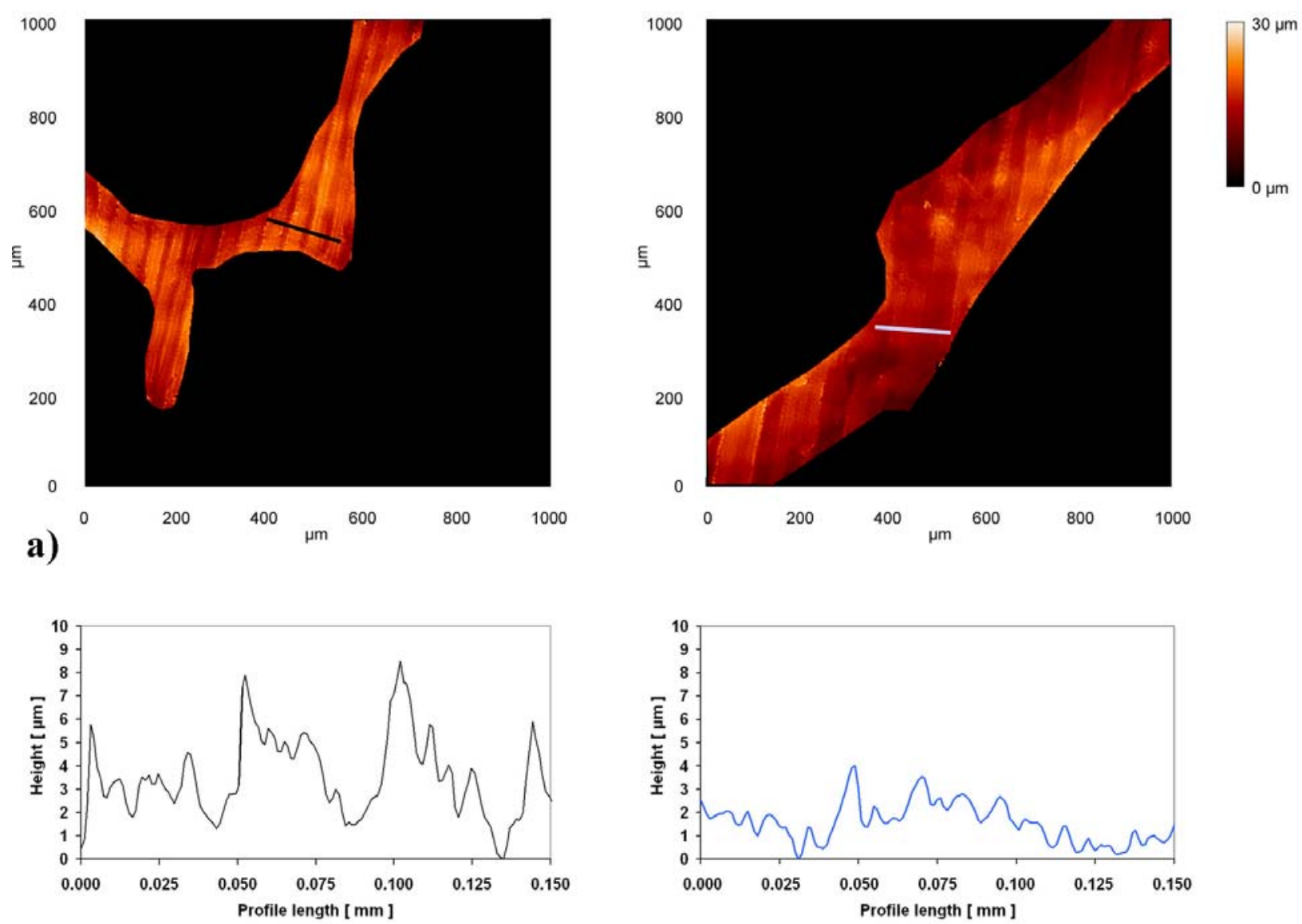

b)

Figure 8. a) Profile analysis of the exposed cut edge was used to compare the Exakt 300 bandsaw and the annular saw. b) It can be seen that the profile from the bone cut with the annular saw is more regular and contains fewer large peaks than the bone cut with the Exakt saw. The largest peak observed with the annular saw was $4 \mu \mathrm{m}$ as opposed to $8 \mu \mathrm{m}$ regularly seen with the Exakt saw.

stain solution while the piston was lifted off the bone surface. A more homogeneous distribution of stain was observed compared to when the piston was in close contact (fig 10b), comparable to that seen in fig 10e, where a bone core was incubated in a beaker of staining solution allowing free diffusion over every surface. Figure $10 \mathrm{f} \mathrm{demonstrated}$ full diffusion of the stain after $13 \mathrm{~h}$ of perfusion where piston and bone surfaces were in tight contact. When the contact was not tight, full perfusion could be seen at around 6 h.

Procion Red: Compared to the results observed with the disulphine blue stain $(566 \mathrm{Da})$, the infiltration of procion red (400 Da) seemed to be less for the same amount of time and flow conditions (fig 11 a,b,c,d). Intense staining of the upper bone surface where contact with the piston was removed before the test could be observed (fig 11e). A transverse section through the middle of a bone core after bathing the core for $3 \mathrm{~h}$, showed a very low intensity of staining.

A perfusion holding system: Using DMEM media $(\mathrm{r}=0,981 \mathrm{~kg} / \mathrm{l}, \mathrm{h}=0,25 \mathrm{~m})$ a pressure of $2405 \mathrm{~Pa}$ was built up over the bone, after both $1 \mathrm{~h}$ and $12 \mathrm{~h}$ no liquid/media flow through the bone was observed (fig 11). As this pressure is much greater than which occurs within the chamber, it would seem to indicate that penetration of the dye into the bone core is as a result of diffusion as opposed to perfusion.

\section{Tissue morphology}

Serial sections of the same tissue were used to compare the stains giemsa, Masson Goldner, Movat pentachrome and toluidine blue embedded within Technovit $9100 \mathrm{New}$ ( $)$. An overview of a cross-section from freshly fixed human cancellous tissue (70-year-old, male) can be seen in figure 12 . The blue square represents the location of the magnified serial section images, depicting calcified ECM and haemopoietic cells, seen below the overview. Since Technovit is not often used with these stains we will describe the colouring observed. Tissue in Figure 12a was stained with giemsa. It stained the ECM purple-pink, and the cells a various shade of blue, purple and pink. It also stained calcified cartilage dark blue, osteoid seams pale blue, nuclei dark blue and erythrocytes pink. This neutral stain is a mixture of two dyes; methylene blue (and its derivatives) as well as eosin (and its derivatives). This dye 


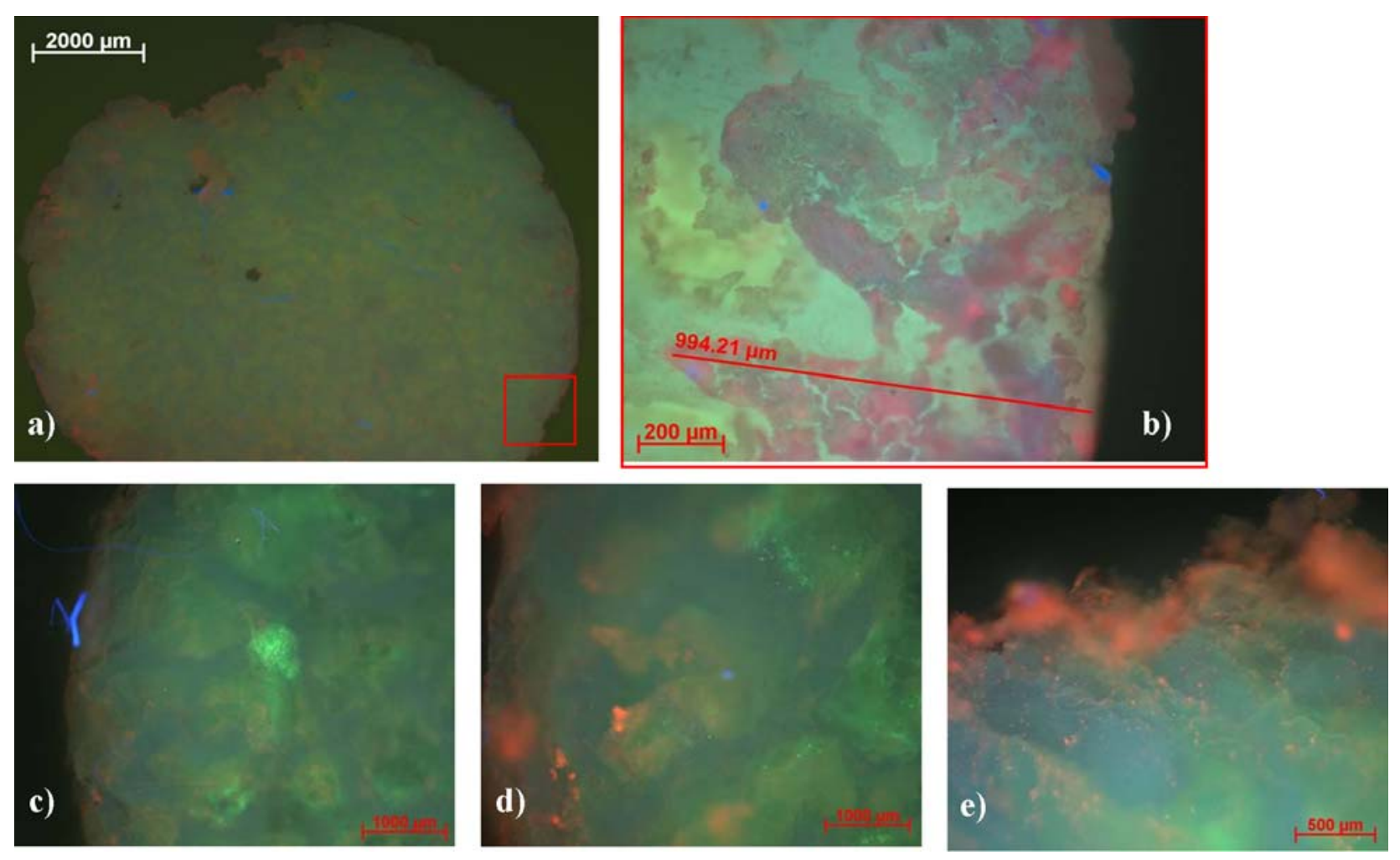

Figure 9. Bovine bone core stained for viability ( $\mathrm{a}$ and $\mathrm{b}$ after fixation in $70 \%$ ethanol) before embedding. a) Overview b) Distance of dead cells from the periphery of the core. c) Overview of a non-fixed bovine bone core. d) Closer view of the same area. e) Closer view of the bone core periphery. Trabeculae are grey, while living cells were labelled green and dead cells labelled red. Due to core thickness single cells were not visible.

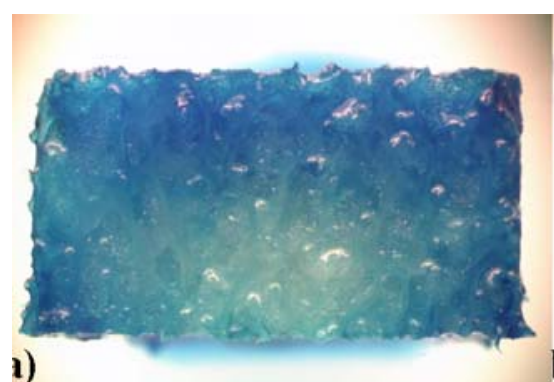

1)

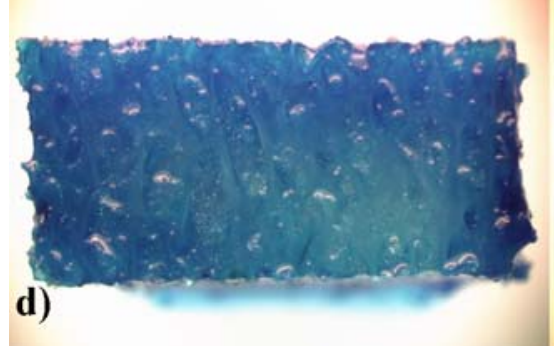

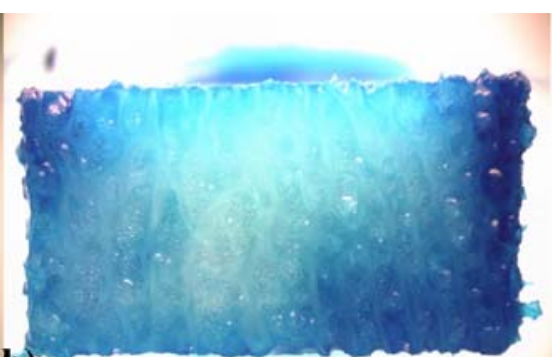

b)

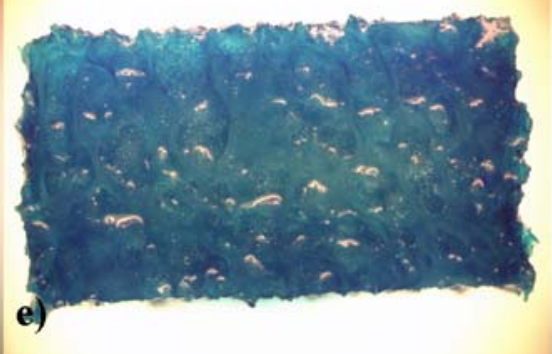

c)
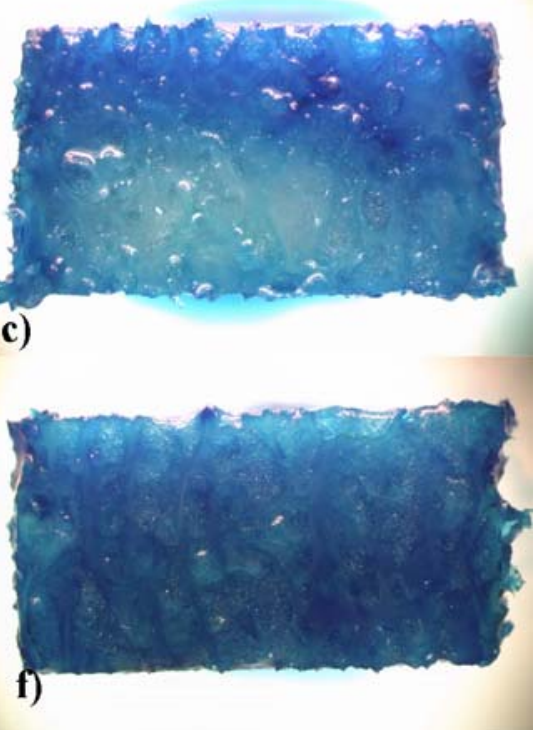

Figure 10. Cross sections through the centre of bovine bone cores incubated for $3 \mathrm{~h}$ with media containing $10 \%$ disulphine blue at various flow rates. a) Flow rate of $7 \mathrm{ml} / \mathrm{h}$. b) Flow rate of $15 \mathrm{ml} / \mathrm{h}$, with clear non-staining where the piston was in close contact above the core. c) Flow rate of $35 \mathrm{ml} / \mathrm{h}$. d) Flow rate of $7 \mathrm{ml} / \mathrm{h}$ with the piston raised from the bone surface. e) A cross section through the centre of a bone core incubated for $3 \mathrm{~h}$ in a beaker of media containing 10\% disulphine blue. f) A bone core incubated for $13 \mathrm{~h}$ at a flow rate of $7 \mathrm{ml} / \mathrm{h}$. 


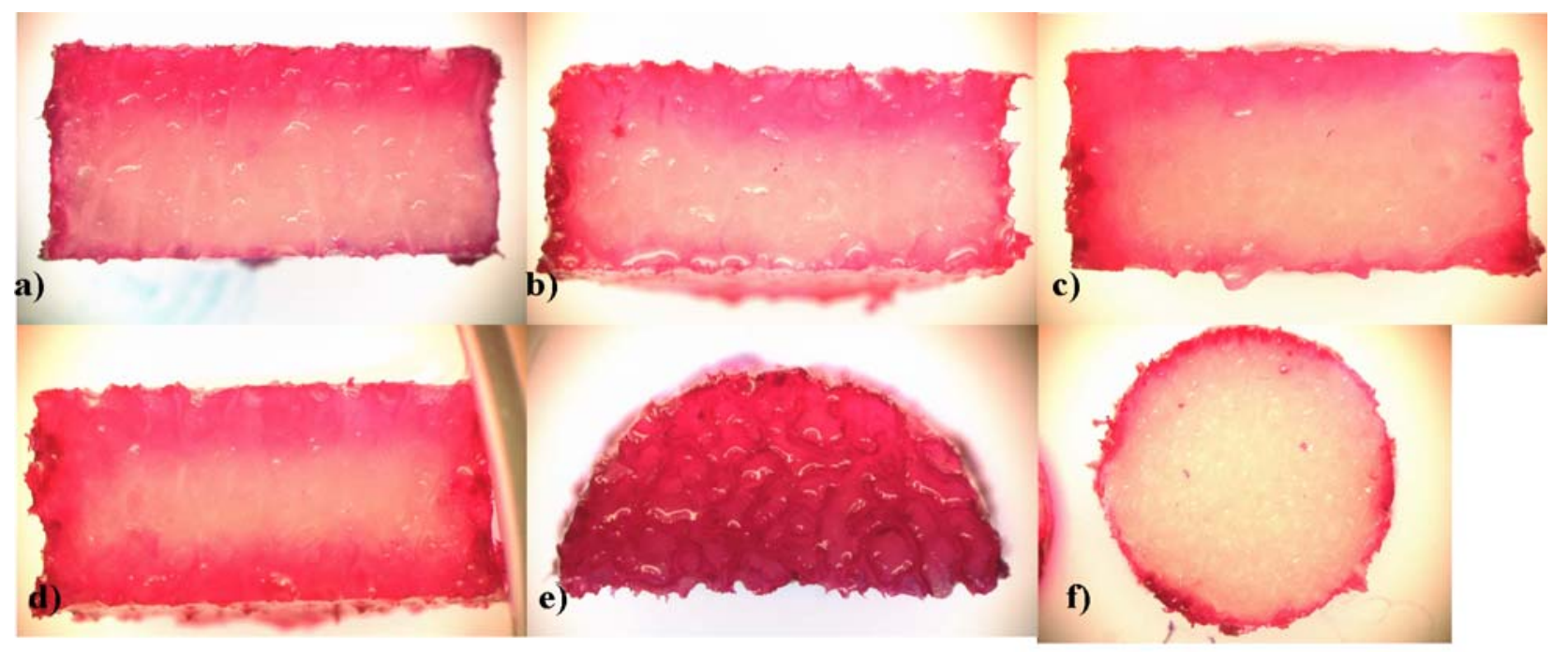

Figure 11. a) A cross section through the centre of bovine bone cores incubated for $3 \mathrm{~h}$ with media containing $10 \%$ procion red at various flow rates. a Flow rate of $7 \mathrm{ml} / \mathrm{h}$. b) Flow rate of $15 \mathrm{ml} / \mathrm{h}$. c) Flow rate of $35 \mathrm{ml} / \mathrm{h}$. d) A cross section through the centre of a bone core incubated for $3 \mathrm{~h}$ in a beaker of media containing $10 \%$ procion red. e) The top surface of bone core at a flow rate of $7 \mathrm{ml} / \mathrm{h}$ with the piston raised from the bone surface. f) A transverse section through the centre of a bone core incubated for $3 \mathrm{~h}$ with media containing $10 \%$ procion red at a flow rate of $7 \mathrm{ml} / \mathrm{h}$.

stains haemopoietic cells (fig 13a), and osteoid seams (fig 14a). Giemsa is particularly suited for staining haemopoietic cells. It is used routinely in hospitals to stain cells in blood smears. Unfortunately it is not so good at low magnifications, and also not suitable for staining osteoclasts. Osteoclasts are often seen as dark blue blobs and the nuclei are difficult to observe.

Figures 12b, 13b and 14b, are all stained with the Masson Goldner stain (Goldner, 1938) a variant of the Masson stain (Masson, 1929). This method is more complex than that of the giemsa, with several stages to achieve the trichrome staining. It characteristically stained the ECM green, the osteoid and cells red (sometimes brown-grey), and the nuclei black (calcified cartilage was red). This stain is recommended for highlighting osteoclasts. It is also used to differentiate calcified tissue and osteoid, though at times the red colour can be lost from the tissue. This is clarified by comparing to MMA embedded tissue (fig 15f). It is possible that the Technovit 9100 New resin may affect the chemical properties of the tissue.

Movat pentachrome is a staining technique that can stain tissue components one of five colours. Its characteristic staining pattern dyes nuclei blue-black, cytoplasm red, osteoid red, connective tissue bright yellow and mineralised cartilage blue-green. This can be seen in figures $12 \mathrm{c}, 13 \mathrm{c}$, and $14 \mathrm{c}$. It works in a similar manner to the Masson stain. This stain is used to differentiate calcified matrix from the osteoid. It also stains haemopoietic cells and osteoclasts much better than giemsa and toluidine blue. However, due to its complex staining procedure the tissue becomes damaged easily and often soft tissue is lost or the calcified matrix is broken.

The final dye used to stain the tissue serial sections, was toluidine blue. This is a basic dye used in an alkaline solution. It stains all bone tissue components various shades of blue when the dye is in its orthochromatic phase. This stain has a unique property known as metachromasia the ability to stain constituents of tissue a different colour from the dye itself. In the metachromatic phase toluidine blue will stain cartilage tissue a purple-red. For example, closely packed sulphate groups found in polysaccharides stain red-purple. This stain provides minimal specific chemical information about the tissue. On the positive side it is possible to observe the mineralisation fronts between the osteoid and the calcified matrix. It is also the stain which preserves tissue to the greatest extent.

\section{Analysis of Core Preparation - Debris}

When comparing the circumference of the bone core with the upper and lower surfaces it became apparent that the circumference of the bone core contained bone debris not seen on the upper or lower surfaces. This would suggest that the drilling protocol used produced bone debris at the periphery of the explant whereas the annular saw did not produce as much (fig 16). Cores were analysed either crosssectionally to get an image of the tissue from each surface including the centre of the core, as well as being analysed transversally to analyse the outer surface of the explants. The debris was more pronounced at the regions of the explant cut with the fluted drill bit, with much less debris observed at the upper and lower surfaces cut with the annular saw (fig 16). The penetration of debris reached as much as $1 \mathrm{~mm}$ into the core.

Complications caused by drilling bone cores, during procedures such as mosaicplasty, have already been documented for both bone and cartilage (Fuchsberger, 1988; Huntley et al., 2005). The core surface area did not 

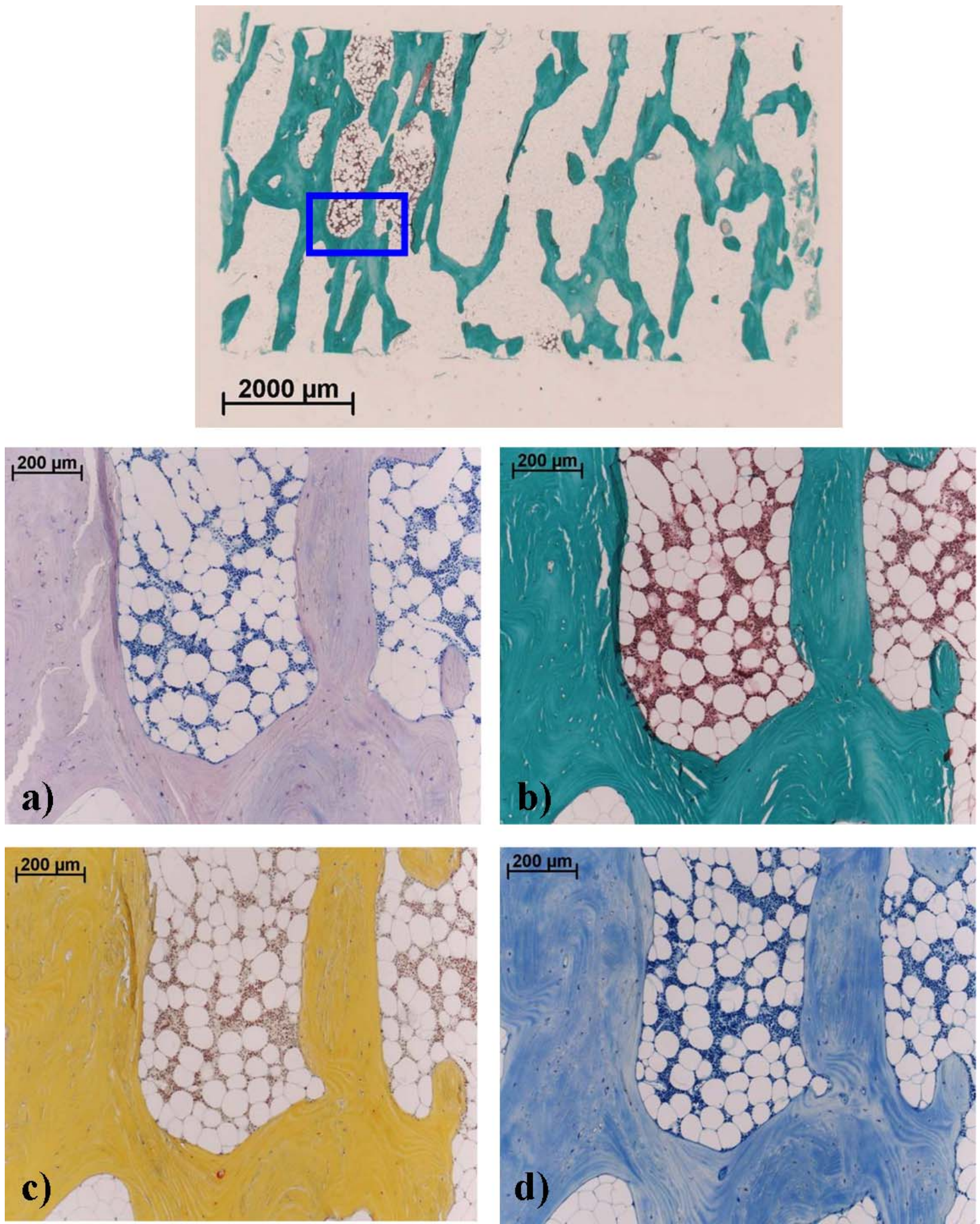

Figure 12. Cross-sectional overview of a section from freshly fixed human tissue (70-year-old male). Blue square represents location of magnified images. a) Giemsa stained serial section. b) Masson stained serial section. c) Movat stained serial section. d) Toluidine blue stained serial section. All depict calcified trabecular matrix, adipocytes and haemopoietic cells. [Characteristic images from one of twenty sections obtained from the centre of the core, providing a representation from a group $n=6]$ 

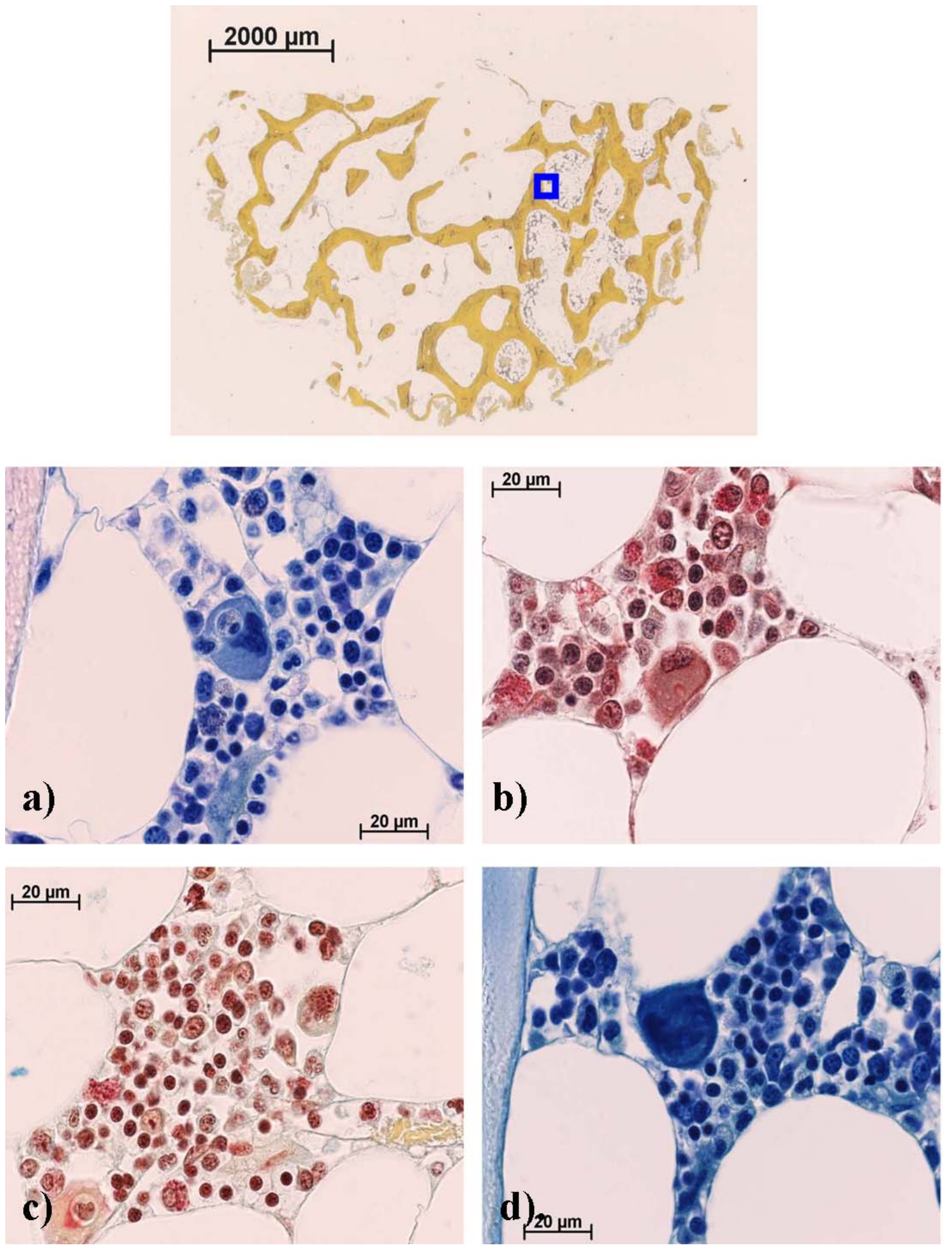

Figure 13. Transverse-sectional overview of a section from freshly fixed human tissue (70-year-old male). Blue square represents location of magnified images. a) Giemsa stained serial section. b) Masson stained serial section. c) Movat stained serial section. d) Toluidine blue stained serial section. All depict haemopoietic cells. [Characteristic image from one of twenty sections obtained from the surface of the core, providing a representation from a group $n=6$ ] 

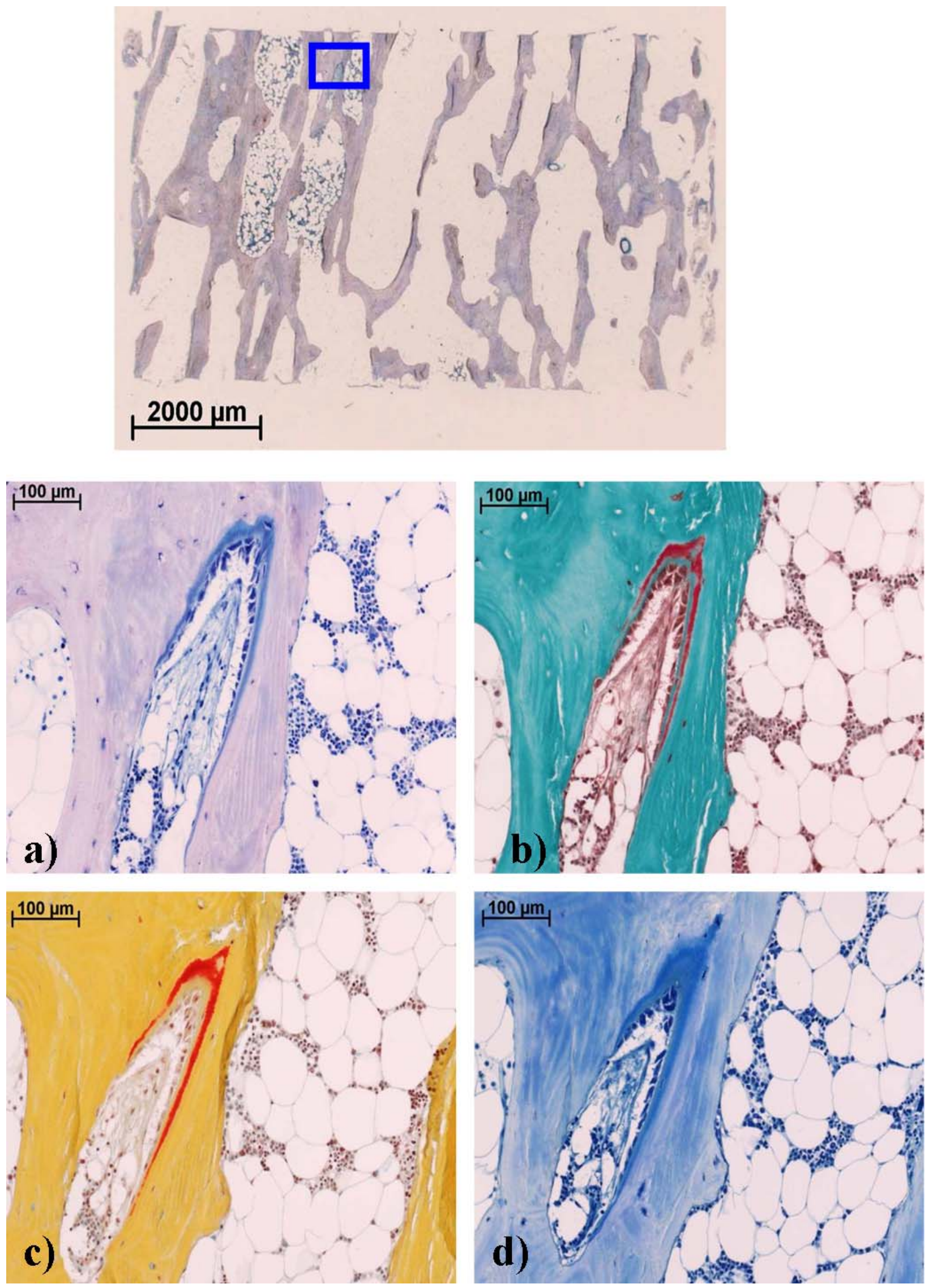

Figure 14. Cross-sectional overview of a section from freshly fixed human tissue (70-year-old male). Blue square represents location of magnified images. a) Giemsa stained serial section. b) Masson stained serial section. c) Movat stained serial section. d) Toluidine blue stained serial section. All depict calcified trabecular matrix, adipocytes and haemopoietic cells and freshly laid uncalcified matrix (osteoid seam). [Characteristic image from one of twenty sections obtained from the centre of the core, providing a representation from a group $n=6$ ] 

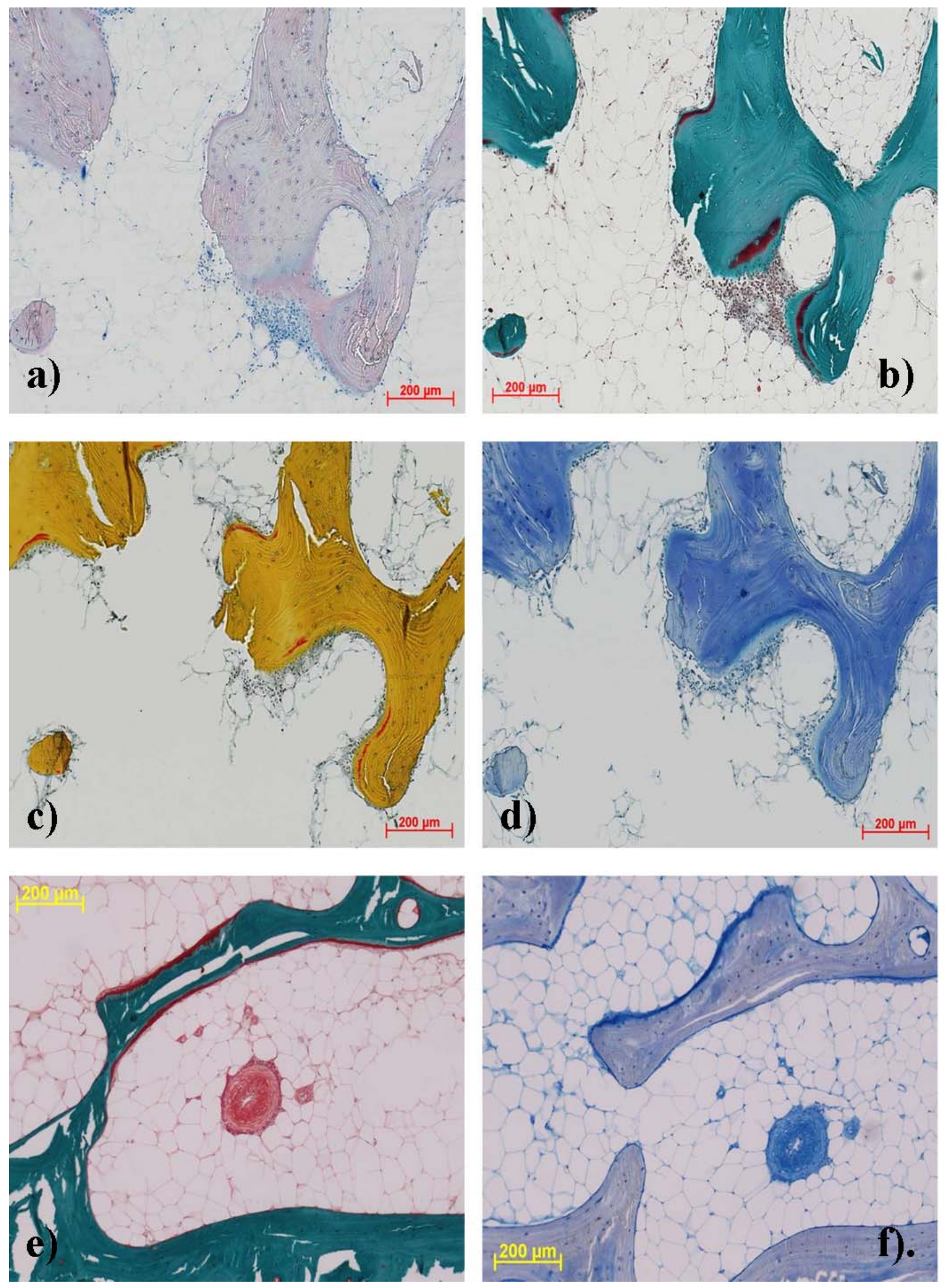

Figure 15. a) Giemsa stained serial section. b) Masson stained serial section. c) Movat stained serial section. d) Toluidine blue stained serial section. All depict calcified trabecular matrix, adipocytes and osteoid seams (bovine tissue, 4-months-old). e) Masson stained MMA embedded section. f) Toluidine blue stained MMA embedded section. Differences in staining pattern was observed. [Characteristic image from one of twenty sections obtained from the centre of the core, providing a representation from a group $n=6$ ] 

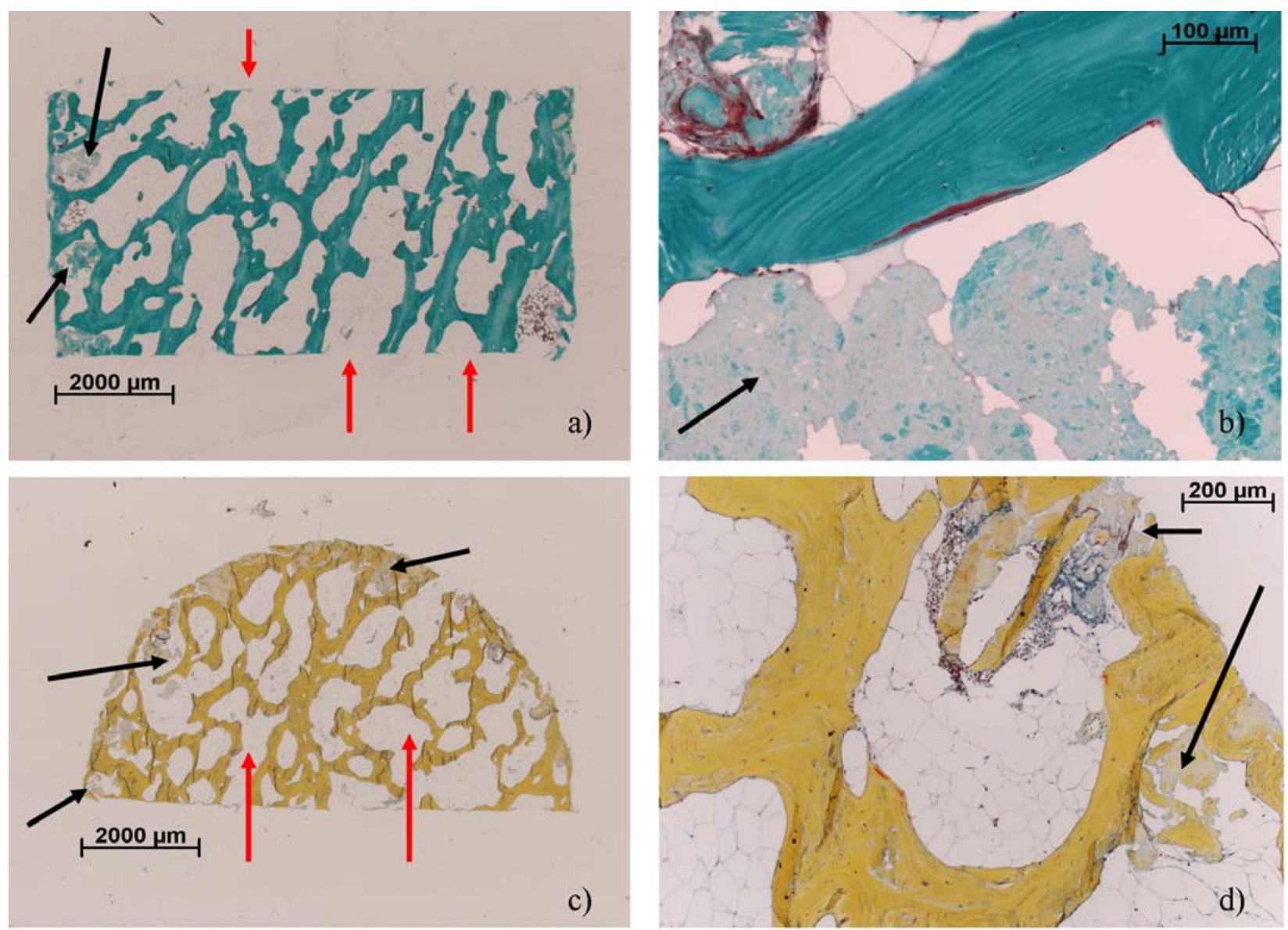

Figure 16. Bone debris observed after fixation of cores directly after drilling and surface cutting a-d. Mason Goldner stained a, b, Movat stained histological sections c, d human tissue, male 71 years). a) Cross-section through the centre of the bone core showing debris at the edges where the core had been drilled (black arrows), but not at the upper or lower surface (red arrows) where the cores had been cut with the annular saw. b) Higher magnification of a) displaying the debris is composed of mineralised tissue. c) Transverse-section of the surface of the bone core showing debris at the edges where the core had been drilled (black arrows), but much less at the upper surface where the cores had been cut with the annular saw (red arrows). d) Higher magnification of c) showing intact adipose tissue after drilling and cutting within the trabecular spaces, plus some areas of bone debris (arrows) at the drill cut edge.
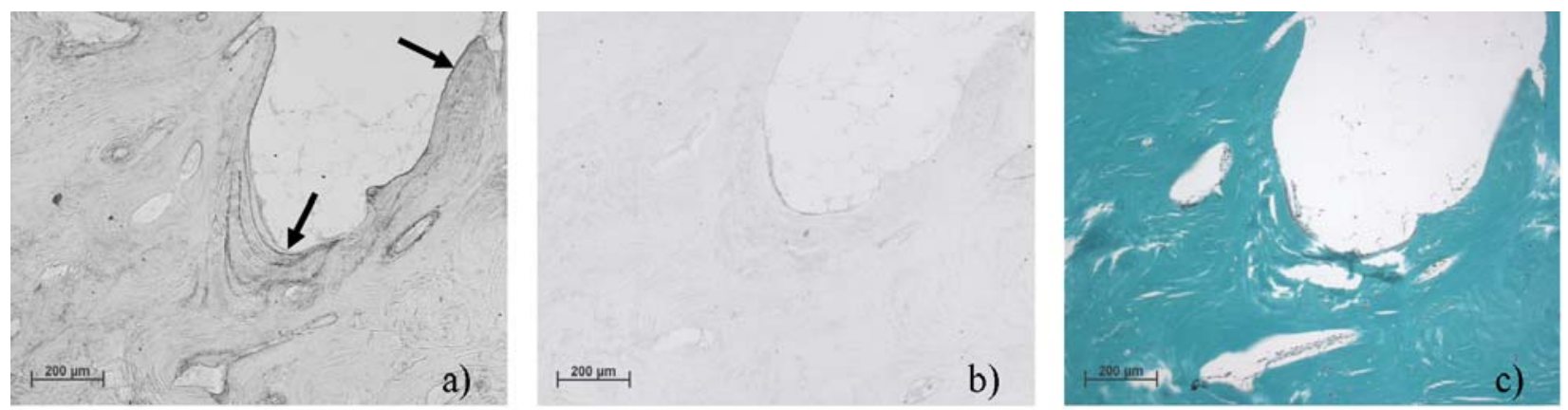

Figure 17. Serial sections from 71 year old human bone samples showing osteopontin labelling (a), negative control (b) and corresponding Masson Goldner staining (c). Staining for osteopontin was most intense on open surfaces where osteoblasts can be found (arrow). 
show as much cell death as there was at the periphery around the circumference of the cores. This effect may be due to less irrigation of the bone core whilst drilling compared to sawing with the annular saw, thus producing more heat resulting in increased thermal necrosis.

\section{Immunolabelling}

Sections immunolabelled with osteopontin demonstrated intense staining corresponding to regions where osteoblasts could be observed (fig 17). This would indicate that cores processed in this way still possess immunological markers expected within bone.

\section{Discussion}

A novel ex vivo mechanically loading culture system for 3D cancellous bone tissue (fig 1 and 2) has been developed to bridge the gap between cell culture and in vivo animal studies. (Jones et al., 2003). During culture a pump is required to move culture medium from the culture tubes to the loading chambers, which are placed on the Zetos loading platform. Force and/or compression can then be applied and measured in real time with high precision $(+/$ $-3 \%$ ) during the application of a variety of different wave forms and frequencies which mimic in vivo conditions. The physiochemical environment can be monitored and controlled. The Zetos system and chambers are housed in a $37^{\circ} \mathrm{C}$ room for the duration of the study. The system has potential in pre-testing the integration of bone with biomaterials, studying basic bone biology, bone diseases and defects e.g. in osteoporosis. While all culture systems have limitations the Zetos system permits the maintenance of a viable $3 \mathrm{D}$ core, which may be loaded and/or treated with a variety of biochemical interventions. The $3 \mathrm{D}$ bone core has limitations in that by maintaining the bone marrow in the cores, there are more than one cell population that may limit the interpretation of the data obtained from the bone core, unlike cell culture where you may look at the specific response of a single cell line. The cores also have no nerve supply, thus no neural factor that may be important in bone response to varied stimuli. However cell culture does not permit the study of ECM response to mechanical and chemical stimuli. There are a number of studies that can be conducted in the Zetos system that cannot be conducted in the animal. One example is the function of the calcium channels that play an important role in the osteoblast and osteocyte response to parathyroid hormone. Blocking these channels in the animal model would result in the death of the animal, thus the only way to follow calcium channel control in bone metabolism is to use the 3D model.

In order to conduct balanced studies in which one does not bias the intervention groups, the investigator must understand and first determine how the trabecular bone tissue is distributed within the bone from which the samples are obtained. The Maceration and Micro-computer Tomography clearly defined the distribution of trabecular bone for each of the areas were the samples were excised. The aim of this study was threefold i) to use a number of different techniques in order to gain a better understanding of cancellous tissue and to optimise the method to analyse tissue before harvest and maintenance in the Zetos culture system; ii) to observe diffusion within the cores within the chambers to determine flow rates for the chambers; iii) to describe the histology and immunohistochemistry of the bone after undergoing biopsy and preparation for culture.

The surfaces of the bone must be plane parallel in order to clearly define the apparent modules of the whole trabecular bone core and then monitor morphological and structural changes. The diffusion of medium into the bone cores is clearly a current limitation of the system, as indicated in the diffusion studies. It must still be determined which method of cutting the final bone core prior to placing them in the chamber will best facilitate diffusion of medium into the core. If one uses the annular saw which results in a roughness of about $4 \mu \mathrm{m}$ versus the band saw which produces a roughness of approximately 8 $\mu \mathrm{m}$, a variation in apparent modulus may occur. However with the $4 \mu \mathrm{m}$ roughness it seems that a vacuum occurs between the bone core and the pistons resulting in minimal or no diffusion under the piston. However with the $8 \mu \mathrm{m}$ of roughness produced by the band saw the parallel surfaces of the bone core may be compromised. Future tests comparing the two methods of preparing the bone sample must be investigated to determine the most effective with respect to diffusion. To counter the variations in roughness, it was decided to use a preload of $10 \mathrm{~N}$ plus 5 $\mu \mathrm{m}$ (dependent on individual sample stiffness), to prevent loss of reproducibility (Jones et al., 2003).

Bone core sizes were chosen for the number of cells present, approximately 2 million, for biochemical analysis (Burger, 2001). Also, the minimum size core required for biomechanical testing would be $7.5 \mathrm{~mm}$ diameter and 6.5 mm high (Linde, Hvid and Madsen, 1992). Despite all the processing required to prepare the cores, it was demonstrated that the retention of marrow within the cores is achieved (fig 12-15) and the whole bone is kept intact. For studies requiring the complete complement of bone derived cells this is an advantage and maintains the bone nearer the in vivo state. The histological methods described will also be valuable tools to investigate the properties of cultured bone cores.

Radiographs taken from a number of bone samples showed that ovine tissue was susceptible to cysts (as was human bone) within the cancellous bone tissue, and was relatively heterogeneous regarding trabecular density. Bovine tissue on the other hand was quite the opposite. It had a very homogeneous distribution of trabeculae throughout its entirety and did not show any sign of cyst formation. This aspect would suggest that the bovine metacarpal tissue would be good for culture in the Zetos system, as samples would be more comparable to each other but care should be taken not to include bone slices which contain the growth plate. We determined that three $7 \mathrm{~mm}$ thick slices provides a large number of bone cores (up to 20) without penetrating the growth plate. Young bovine material is also likely to react more rapidly to any mechanical signals as the tissue is quite active. Older tissue with a slow turnover rate will require more long term 
experiments to make any differences apparent. However, it is known from the literature that trabecular bone from skeletally immature animals (such as that seen in the bovine model) is weaker, less stiff, more deformable before failure; and, has higher shock-absorptive qualities than skeletally mature animals (Nafei, Danielsen, Linde and Hvid, 2000). Thus, it is not only the bones' apparent density that causes variations in the compressive elastic properties of cancellous tissue but collagen content and the mineral content are also major predictors. This should be taken into consideration when selecting tissue for culture in the Zetos system.

Limitations of this technique is that plain film radiography does not record the real architecture of bone, but an apparent one which is created by superposition of the X-ray shadows produced by calcified tissues. Thus, in order to get non-artefactual results, each bone section requires the same thickness throughout its entirety. The quality of the radiographs may also be affected by the presence of soft tissue on the bone's outer surface, which can cause blurred images. Finally, radiography only gives a $2 \mathrm{D}$ view of the tissue and says nothing of the quality or connectivity through the volume of the section. Though this technique is not perfect, it does have potential to be used prior to explant harvest, in order to assess the quality of the tissue qualitatively and to show areas to avoid harvesting from, if the technique can be shown not to harm the cells.

An advantage over radiography would be a technique that showed the 3D structure of cancellous bone tissue. This is possible with the microCT. The availability of 3D measuring techniques and 3D image processing methods allow direct quantification of unbiased morphometric parameters, such as volume and surface area. MicroCT allows the capture of true trabecular architecture without assumptions of the structure type. The results from this analysis confirmed that seen with radiography, indicating that ovine and human tissue were more heterogeneous than the bovine tissue. MicroCT is advantageous in that fact that, unlike radiography, it can measure connectivity. The $3 \mathrm{D}$ connectivity of a sample is defined as the degree to which a structure is multiply connected. Changes in connectivity are associated with changes in elastic stiffness, though the absolute measure of connectivity is not directly related to mechanical strength (Kinney and Ladd, 1998). There is no functional relationship between connectivity and elastic modulus per se because connectivity does not discriminate between rod-like connections and fenestrated plates.

The microCT results obtained for the architecture of individual bone cores harvested from ovine, bovine, and human tissues were generated using the "Plate Model". This method assumes all trabecular bone was organised in infinite parallel plates. These results are, therefore, only valid under the assumption of a plate model. The results would be biased if all trabecular bone was not organised in parallel plates and this is a limitation to using modelbased approaches (Kinney et al., 1995). Currently microCT is not suitable in clinical settings as it has a high radiation dose and only possible to generate a 3D image with small biopsies. If it can be determined however, that the microCT method does not negatively influence the bone cells phenotype or viability, microCT images taken pre- and post- culture would be invaluable to calculate where and how much bone was resorbed and deposited ex vivo. The resolution may be a little low to see differences from shortterm cultures. The effect of microCT on the biological activity of bone tissue must be further investigated. Protocols are currently being developed to evaluate the impact of microCT on bone core viability.

Characterisation of ovine, bovine and human tissue with radiography and microCT confirmed previous findings that there was a vast difference between cancellous bone density within the same animal at the site of removal (Whitehouse, Dyson and Jackson, 1971; Whitehouse and Dyson, 1974), which was dependent on age, species, and location of individual explants within the region of choice. A limitation regarding cancellous tissue location for these studies is the amount of cores that can be harvested from a particular area. The way that this effect may have influenced cancellous bone cores cultured in the Zetos system is outlined with microCT images of the bone cores in figure 7.

Goldstein (1987) analysed the mechanical properties of human tibial trabecular bone as a function of metaphyseal location. His results indicate that the medial side of the bone is stronger and stiffer than the lateral. This correlated with the belief that bone is stronger and stiffer directly beneath load bearing areas. Hobatho et al. (1997), analysed specimens of human cancellous bone from the tibia, femur, patella, lumbar spine, and humerus of eight different subjects. The elastic properties of these bone explants differed significantly $(\mathrm{p}<0.05)$ between subjects and the mechanical properties varied along the length and periphery. These observed variations were shown to be a function of anatomical position, loading direction, methods of storage and testing conditions. Similar observations were confirmed with the Zetos system through a series of loading events on ovine and bovine cancellous bone explants (Unpublished data). These results indicate that when using bone cores in which a number of group interventions are to be conducted, one must use a block design based on the apparent modulus of the bone cores as an underlying principle for group formation.

To accurately measure sample stiffness, the surface of the sample to be measured needs to be parallel, as only a perfect plane parallel body will follow Hooke's law (though such a surface does not really exist) and so both the Exakt saw and annular saw were investigated. If the surfaces are not parallel then the effective area under load increases as the force increases, thus, a function of force over compression will be non-linear. If the surfaces are parallel but rough, then the curve will be linear after a short initial non-linear phase. However, there does remain an inaccuracy about the effective area, which may lead to an incorrect Young's modulus. In order to obtain precise results great care is needed in preparing the samples to be tested. In order to help eliminate the problem of surface parallelism, it is important to bring all surfaces in contact with one another. To achieve this, a small preload is placed 
upon the chamber $\sim 2.5 \mathrm{~N}$ prior to loading, thus the surface of the piezo actuator is in contact with the piston, which is in turn in contact with the bone core, the under side of the bone core is in contact with the base plate and this in turn in contact with the bottom of the loading device. Data is usually recorded daily for the duration of the experiment, though the readings for the first day are excluded due to artefacts caused by surface roughness. It is not possible to determine absolute stiffness due to the machining of the sample core biopsies. Trabecular struts that were once supported are now cut and this leads to a platen effect, where bone faces are misaligned with the loading platen (piston and base plate). Thus, data obtained from this system cannot be extrapolated to the whole bone tissue. Cutting of the cores causes an "end effect" due to the machining of the specimen. Trabeculae at the boundary of the core are unsupported, thus strain is greater at the edge of the specimen than in the middle. Due to an elevated strain at the end of the specimen in contact with the loading platen an overestimation of strain may cause an underestimation of Young's modulus (Odgaard et al., 1989). The use of bone cements would greatly reduce this effect, by stabilising the cut ends, but due to the culture constraints of this system it would greatly affect the diffusion of medium from reaching bone cells within the explant and equally removal of waste from the explant.

\section{Conclusion}

In conclusion, cancellous bone is dramatically heterogeneous and great care should be taken to get representative and reproducible results. There is inter-site and inter-individual variability. Caution is required when extrapolating data obtained from one model to other models and to the situation in vivo. While this data on the preparation of the tissue for experimentation was collected specifically for use with the Zetos loading machine, many of the conclusions are equally valid for the source and preparation of tissue to be used in any experiments involving the mechanical loading of bone cores. The loading and media diffusion is believed to be more physiological than current organ culture techniques, which suffer from oxygen partial pressure problems and very poor diffusion of nutrients through the cortical bone. In the ensuing second paper we describe histology, immunohistochemistry, biochemistry and viability studies of the cultured samples and compare these to the time zero samples (directly after core biopsy and preparation for culture). Several problems and remedies as well as pitfalls will also be detailed and discussed.

\section{Acknowledgements}

The authors wish to thank 3R (grants \# 78/01 and 83/03) for their continuous financial support for this work. Thanks also to European space agency (ESA) Microgravity Application Programme (MAP) (grant \#AO-99-122) for partial financial support. Thanks to Dr Thomas Perren,
Davos hospital for supplying human tissue. Thanks to Pamela Furlong (ARI) for general technical assistance with preparation, culturing and histology of the cores and Marita Kratz, Eckhardt Broekmann and Thorsten Pohl (Marburg) for original training with the machine for operating and culturing. Thanks also to Christoph Sprecher for technical assistance with the confocal microscope, Christina Eckhardt for technical assistance with the microCT.

\section{References}

Burger EH (2001) in Bone Mechanics Handbook, Ed. Cowin SC. CRC Press, New York, Chapter 28, pp 1-16.

Fell HB, Robinson R (1929) The growth development and phosphatase activity of embryonic avian femora and limb-buds cultivated in vitro. Biochem J 23: 83-87.

Fuchsberger A (1988) Damaging temperature during the machining of bone. Unfallchirurgie 14:173-183.

Goldner J (1938) A modification of the Masson Trichrome technique for routine laboratory purposes. Am J Pathol 14: 237-243.

Goldstein SA (1987) The Mechanical Properties of Trabecular Bone: Dependence on Anatomical Location and Function. J Biomechanics 20: 1055-1061.

Hobatho MC, Rho JY, Ashman RB (1997) Anatomical variation of human cancellous bone mechanical properties in vitro. Stud Health Technol Inform 40: 157-173.

Huntley JS, McBirnie JM, Simpson AH, Hall AC (2005) Cutting-edge design to improve cell viability in osteochondral grafts. Osteoarthritis Cartilage 13: 665-671.

Jones DB, Broeckmann E, Pohl T, Smith EL (2003) Development of a mechanical testing and loading system for trabecular bone studies for long term culture. Eur Cell Mater 5: 48-60.

Kinney JH, Lane NE, and Haupt DL (1995) In vivo, three-dimensional microscopy of trabecular bone. J Bone Miner Res 13: 839-845.

Kinney JH, Ladd AJC (1998) The relationship between three-dimensional connectivity and the elastic properties of trabecular bone. J Bone Miner Res 10: 264-270.

Linde F, Hvid I, Madsen F (1992) The effect of specimen geometry on the mechanical behaviour of trabecular bone specimens. J Biomech 25: 359-368.

Lozupone E, Palumbo C, Favia A, Ferretti M, Palazzini S, Cantatore FP (1996) Intermittent compressive load stimulates osteogenesis and improves osteocyte viability in bones cultured "in vitro". Clin Rheumatol 15: 563-572.

Masson P (1929) Trichrome stainings and their preliminary technique. J Tech Meth 12: 75-90.

Meghji S, Morrison MS, Henderson B, Arnett TR (2001) pH dependence of bone resorption: mouse calvarial osteoclasts are activated by acidosis. Am J Physiol Endocrinol Metab 280: E112-E119.

Nafei A, Danielsen CC, Linde F, Hvid I (2000) Properties of growing trabecular ovine bone. J Bone Surg Br 82: 910-920.

Odgaard A, Hvid I, Linde F (1989) Compressive axial strain distributions in cancellous bone specimens. J Biomech 22: 829-835. 
Rawlinson SC, Mosley JR, Suswillo RF, Pitsillides AA, Lanyon LE (1995) Calvarial and limb bone cells in organ and monolayer culture do not show the same early responses to dynamic mechanical strain. J Bone Miner Res 10: $1225-2332$.

Spengler DM, Moery ER, Carter DR, Turner RT, Baylink DJ (1983) Effects of space flight on structural and material strength of growing bone. Proc Soc Exp Biol Med 174: 224-228.

Strangeways TSP, Fell HB (1926) Experimental studies on the differentiation of embryonic tissues growing in vivo and in vitro. (1) The development of the isolated limb-bud (a) when subcutaneously grafted into the post-embryonic chick and (b) The development of the isolated early embryonic eye of the fowl when cultivated in vitro. Proc Roy Soc B99: 340-364.

Vico L, Chappard D, Alexandre C, Palle S, Minaire P, Riffat G, Morukov B, Rakhmanov S (1987) Effects of a 120 day period of bed-rest on bone mass and bone cell activities in man: attempts at countermeasure. Bone Miner 5: 383-394.

Whitehouse WJ, Dyson ED, Jackson CK (1971) The Scanning Electron Microscope in Studies of Trabecular Bone from a Human Vertebral Body. J Anat 108: 481496.

Whitehouse WJ, Dyson ED (1974) Scanning Electron Microscope studies of Trabecular Bone in the Proximal End of the Femur. J Anat 118: 417-444.

Yang R, Davies CM, Archer CW, Richards RG (2003) Immunohistochemistry of matrix markers in Technovit $9100 \mathrm{New}^{\circledR}$ - embedded undecalcified bone sections. Eur Cell Mater 6: 57-71.

\section{Discussion with Reviewers}

V Salih: Can the authors be sure that the higher concentrations of antibiotic/antimycotic used did not affect cellular metabolism and/or function prior to experimental analyses?

Authors: We also would prefer to use lower concentrations of antibiotic and antimycotic, in fact recent work has been carried out without the use of Fungizone (the more toxic of the reagents) with no increase in contaminations. Unfortunately, due to the source of tissue and its three dimensional nature, we believe that an antibiotic wash is a necessary evil to ensure a sterile environment. That said we appreciate it may lead in the short term to a general depression of protein synthesis.

P Prendergast: The authors are to be congratulated on further development of the Zetos system. It has the potential to be a definite step forward in our ability to investigate bone biomechanics and mechanobiology. Clearly the potential of mechanically loading bone whilst maintaining cell viability and conducting online monitoring of properties could produce important advances. One technical question is: was it ever considered to incorporate it into a microCT scanner so continuous monitoring of new bone formation could be recorded? Is such a thing even possible?

Authors: Exactly this issue is currently being investigated by our collaborators. The aim is to design a loading chamber which can be placed within a $\mu \mathrm{CT}$ scanner.

P Prendergast: The paper presents mostly material that is preparatory to using the Zetos system to test hypotheses in bone mechanobiology, and finishes with a thorough discussion of the issues involved. One of the crucial issues is perfusion of nutrients to the cells in the bone core. Does the presence of dead cells with the core (as indicated in Fig. 8) not pose problems for the Zetos system, and for its potential to conduct long term studies?

Authors: We are currently investigating ways to reduce the cell death cause during the drilling of the cores. Treating the cores with apoptosis inhibitors is one way we believe this cell death can be reduced. 